\title{
Alternating Direction Implicit Methods for Parabolic Equations With a Mixed Derivative
}

Richard M. Beam and R. F. Warming

March 1979

\section{LBRTN BOPI] \\ Bद? $79 / 9$}

LANGiey RESEARCH CENTER

LIERARY, NASA

HAMPTON, YIRGINA

\section{N/Sก}

National Aeronautics and 


\section{Alternating Direction Implicit Methods for Parabolic Equations With a Mixed Derivative}

Richard M. Beam

R. F. Warming, Ames Research Center, Moffett Field, California

\section{NNSA}




\title{
ALTERNATING DIRECTION IMPLICIT METHODS FOR PARABOLIC
}

EQUATIONS WITH A MIXED DERIVATIVE

\author{
RICHARD M. BEAM ${ }^{\dagger}$ and R. F. WARMING ${ }^{\dagger}$
}

\begin{abstract}
Alternating direction implicit (ADI) schemes for twodimensional parabolic equations with a mixed derivative are constructed by using the class of all $\mathrm{A}_{\mathrm{o}}$-stable linear two-step methods in conjunction with the method of approximate factorization. The mixed derivative is treated with an explicit two-step method which is compatible with an implicit $A_{0}$-stable method. The parameter space for which the resulting ADI schemes are secondorder accurate and unconditionally stable is determined. Some numerical examples are given.
\end{abstract}

\section{CONTENTS}

1. Introduction ....................... 2

2. Preliminaries .................... 5

2.1. Linear multistep methods . . . . . . . . . . . . 5

2.2. One-leg (multistep) methods . . . . . . . ..... 8

2.3. Combined linear multistep methods . . . . . . . . . . 9 9

3. Unconditionally stable schemes . . . . . . . . . . . . 10

4. An ADI scheme: the $\rho(E)$ or $\Lambda$ formulation ........... 15

5. A general two-step ADI scheme . . . . . . . . . . . . 19

5.1. General formulation . . . . . . . . . . . . . 19

5.2. Special cases of general formulation . . . . . . . . . 21

†Computational Fluid Dynamics Branch, Ames Research Center, NASA, Moffett Field, California 94035. 
5.3 Algorithm selection . . . . . . . . . . . . . . . 24

5.4 General formulation with no mixed derivative . . . . . . . . 26

6. Time-dependent coefficients . . . . . . . . . . . . . 27

7. Numerical examples . . . . . . . . . . . . . . . 29

8. Concluding remarks . . . . . . . . . . . . . . . . . 34

Appendix A: Stability analysis of combined LMMs . . . . . . . . . . 36

Appendix B: Stability analysis for two-step ADI schemes . . . . . . . . 38

References . . . . . . . . . . . . . . . . . 43

1. Introduction. When an alternating direction implicit (ADI) method is applied to a parabolic equation, for example,

$$
\frac{\partial u(x, y, t)}{\partial t}=L u(x, y, t) \text {, }
$$

where

$$
L=a(x, y, t) \frac{\partial^{2}}{\partial x^{2}}+b(x, y, t) \frac{\partial^{2}}{\partial x \partial y}+c(x, y, t) \frac{\partial^{2}}{\partial y^{2}},
$$

$(1.1 c, d)$

$\mathrm{a}, \mathrm{c}>0, \mathrm{~b}^{2}<4 \mathrm{ac}$,

it reduces the computational problem to a sequence of one-dimensional (matrix) inversion problems. If the mixed derivative $\partial^{2} / \partial x \partial y$ of the operator $L$ were absent $(b=0)$, an ADI method would reduce the operator $(1-L)$ to the product of two one-dimensional spatial operators. In the method of Douglas and Gunn [9], the mixed derivative is kept implicit and their scheme requires four inversions; that is, the operator $(1-L)$ is reduced to four onedimensional operators. The two additional inversions are the direct result of keeping the mixed derivative implicit. Simpler and more efficient schemes can be obtained if the mixed derivative is evaluated explicity. Perhaps not so obvious is the stability of these simpler schemes. In fact, it is 
rather surprising that one can develop unconditionally stable algorithms for (1.1) by computing the mixed derivative explicitly and the derivatives $\partial^{2} / \partial x^{2}$ and $\partial^{2} / \partial y^{2}$ implicitly. McKee and Mitche11 [15] surveyed two-level, first-order accurate (in time) schemes and devised a new first-order accurate, unconditionally stable scheme for (1.1). Tyengar and Jain [12] generalized the method of McKee and Mitche11 and presented a three-level, second-order accurate scheme for (1.1); however, the implementation of the scheme is complicated by the explicit computation of fourth differences although the original partial differential equation (1.1) contains only second derivatives.

The present authors constructed a second-order accurate ADI algorithm for the compressible Navier-Stokes equations [1]. When the algorithm is applied to the model equation (1.1), it leads to a simple three-level unconditionally stable scheme which is easy to implement. In a recent paper [20] we combined A-stable linear multistep methods (LMMs) and approximate factorization to construct a large class of multilevel unconditionally stable ADI schemes for a model partial differential equation with both convective (hyperbolic) and diffusive (parabolic) terms; that is,

$$
L=a \frac{\partial^{2}}{\partial x^{2}}+b \frac{\partial^{2}}{\partial x \partial y}+c \frac{\partial^{2}}{\partial y^{2}}-c_{1} \frac{\partial}{\partial x}-c_{2} \frac{\partial}{\partial y},
$$

where $a, b, c$ satisfy $(1.1 c, d)$ and $c_{1}, c_{2}$ are real constants. The general formulation of [20] is second-order accurate if $b=0$ but first-order accurate in time if the mixed derivative is included. In both [1] and [20], the mixed derivative is treated explicitly. The purpose of the present paper is to modify and combine the algorithms of [1] and [20] to obtain a general, second-order accurate, unconditionally stable algorithm for the model parabolic equation (1.1). In a companion paper [21] we apply the method to derive a 
noniterative ADI algorithm for a hyperbolic-parabolic system of nonlinear equations with mixed derivatives.

The development and analysis of numerical methods for ordinary differential equations (ODEs) is more advanced than that for partial differential equations (PDEs). Therefore, it seems plausible to capitalize on this fact by making use of known results from the theory of difference methods for ODEs to construct methods for PDEs. For example, the time differencing schemes used to construct implicit methods for PDEs are invariably LMMs although this fact is seldom noted. Since a great deal is known about the properties of LMMs (see, e.g., $[6,10]$ ), one can use this information to advantage when attempting to construct schemes for PDEs. With these observations in mind we use Sec. 2 as a review of the theory and notation for linear multistep methods including A-stability, $A_{0}$-stability, and one-leg methods. In addition, we introduce the notion of an LMM combining two different LMMs - one implicit and the other explicit. In Sec. 3 we investigate the stability of an implicit method for (1.1) obtained by using an $A_{0}$-stable LMM as the time differencing method. We then modify the scheme by treating the mixed derivative explicitly and reinvestigate the stability properties. The method of approximate factorization is applied in Sec. 4 to obtain an unconditionally stable, second-order accurate, multistep ADI scheme in the $\rho(E)$ formulation. In Sec. 5 we construct a general approximate factorization method for all second-order, two-step schemes and discuss the parameter space for unconditional stability. Details of the stability analyses are given in the Appendices A and B. In Sec, 6 a simple modification is given for the case of time-dependent coefficients $a, b, c$. Numerical examples are given in Sec. 7 and some concluding remarks in Sec. 8 . 
2. Preliminaries. In this section we briefly review the theory of linear multistep methods (LMMs) and the related one-leg (multistep) methods. In addition, we introduce combined LMMs.

2.1. Linear multistep methods. A linear $k$-step method for the firstorder ordinary differential equation

$$
\frac{d u}{d t}=f(u, t), \quad u(0)=u_{0}
$$

is defined by the difference equation

$$
\rho(E) u^{n}=\Delta t \sigma(E) f^{n},
$$

where $\rho$ and $\sigma$ are the generating polynomials,

$(2.3 a, b)$

$$
\rho(\zeta)=\sum_{j=0}^{k} \alpha_{j} \zeta^{j}, \quad \sigma(\zeta)=\sum_{j=0}^{k} \beta_{j} \zeta^{j},
$$

and $E$ is the shift operator, that is,

$$
\mathrm{Eu}^{\mathrm{n}}=\mathrm{u}^{\mathrm{n}+1} \text {. }
$$

In (2.2), $u^{n}$ is the numerical solution at the point $t=t^{n}=n \Delta t, \Delta t$ is the time step, and $f^{n}=f\left(u^{n}, t^{n}\right)$. The method is explicit if $\beta_{k}=0$ and implicit otherwise. Consistency and normalization are expressed by the relations:

$$
(2.5 a, b, c)
$$

$$
\sum_{j=0}^{k} \alpha_{j}=0, \quad \sum_{j=0}^{k} j \alpha_{j}=\sum_{j=0}^{k} \beta_{j}=1 .
$$

As an example of an LMM, the most general consistent two-step method (i.e., $k=2$ in $(2.3)$ ) can be written as

$$
(1+\xi) u^{n+2}-(1+2 \xi) u^{n+1}+\xi u^{n}=\Delta t\left[\theta f^{n+2}+(1-\theta+\phi) f^{n+1}-\phi f^{n}\right]
$$

where $(\theta, \xi, \phi)$ are arbitrary real numbers. The operators $\rho(E)$ and $\sigma(E)$ are

$$
\begin{aligned}
& \rho(E)=(1+\xi) E^{2}-(1+2 \xi) E+\xi, \\
& \sigma(E)=\theta E^{2}+(1-\theta+\phi) E-\phi .
\end{aligned}
$$


For the class of all two-step methods that are at least second-order accurate, the parameters $(\theta, \xi, \phi)$ are related by

$$
\phi=\xi-\theta+\frac{1}{2}
$$

and consequently, $\sigma(E)$ can be rewritten in terms of the two parameters $(\theta, \xi)$ as

$$
\sigma(E)=\theta E^{2}+\left(\xi-2 \theta+\frac{3}{2}\right) E-\left(\xi-\theta+\frac{1}{2}\right) \text {. }
$$

Some well-known implicit second-order methods and their corresponding values $(\theta, \xi, \phi)$ are listed in Table 1 . Linear one-step methods are a subclass of (2.6) obtained by setting $\xi=\phi=0$ :

$$
u^{n+1}-u^{n}=\Delta t\left[\theta f^{n+1}+(1-\theta) f^{n}\right],
$$

where we have shifted the time index down by one. The trapezoidal formula $(\theta:=1 / 2$ in $(2.10))$

$$
u^{n+1}-u^{n}=\frac{\Delta t}{2}\left(f^{n+1}+f^{n}\right)
$$

is the only second-order accurate one-step method. When the trapezoidal formula is applied to a parabolic equation, the resulting algorithm is usually called Crank-Nicolson.

The linear stability of an LMM is analyzed by applying it to the linear test equation

$$
\frac{\mathrm{du}}{\mathrm{dt}}=\lambda \mathbf{u},
$$

where $\lambda$ is a complex constant. The stability is determined by the location of the roots of the characteristic equation,

$$
\rho(\zeta)-\lambda \Delta t \sigma(\zeta)=0,
$$

relative to the unit circle in the complex plane. The stability region of an LMM consists of the set of all values of $\lambda \Delta t$ for which the characteristic equation (2.13) satisfies the root condition; that is, its roots $\zeta_{\ell}$ all 
satisfy $\left|\zeta_{\ell}\right| \leq 1$ and the roots of unit modulus are simple. An LMM is said to be A-stable if its stability region contains all of the left half of the complex $\lambda \Delta t$ plane including the imaginary axis [4]. A simple test for A-stability can be formulated in terms of positive real functions [7]. By applying the test to the linear two-step method (2.6), one finds that the method is A-stable if and only if

$\theta \geq \phi+\frac{1}{2}$,

$(2.14 \mathrm{~b})$ $\xi \geq-\frac{1}{2}$ $\xi \leq \theta+\phi-\frac{1}{2}$

An LMM is said to be $A_{0}-$ stable if the region of stability contains the negative real axis of the complex $\lambda \Delta t$ plane, that is, the interval $(-\infty, 0]$. Again, by applying the theory of positive real functions, one finds that LMM (2.6) is $A_{0}$-stable if and only if
$\theta \geq \phi+\frac{1}{2}$,
$(2.15 b)$
$\xi \geq-\frac{1}{2}$
$(2.15 c)$
$0 \leq \theta+\phi$.

The details of the analysis for obtaining these inequalities are described in [21]. For first-order accurate schemes, the inequality (2.15c) is less stringent than (2.14c). This is not surprising since $A_{0}$-stability is a weaker requirement than A-stability. However, for the class of all second-order methods, the parameters $(\theta, \xi, \phi)$ are related by (2.8), and both sets of inequalities (2.14) and (2.15) reduce to
$(2.16 a, b)$
$\xi \leq 2 \theta-1$,
$\xi \geq-\frac{1}{2}$ 
The parameter space $(\theta, \xi)$ for which the class of two-step, second-order methods is A-stable, happens to coincide with the parameter space for which the class is $A_{0}$-stable and is shown by the shaded region of Fig. 1. The methods listed in Table 1 and indicated by the symbols in Fig. 1 are both $A_{O^{-}}$and A-stable. Dahlquist has proved that the order of accuracy of an A-stable LMM cannot exceed two [4]. On the other hand, Cryer [3] has proved there exist

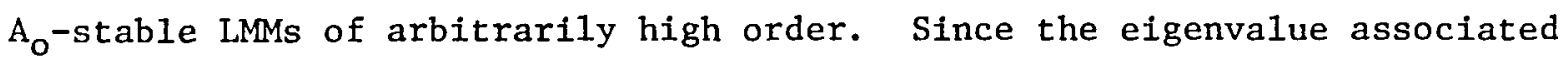
with the parabolic equation (1.1) is real and negative, the application of an $A_{0}$-stable LMM will yield an unconditionally stable scheme, that is, no stability restriction on the size of $\Delta t$.

In this paper we restrict our attention to second-order accurate LMMs. This limitation is motivated by two practical considerations. First, conventional techniques for constructing alternating direction implicit (all A-stable and $A_{0}$-stable LMMs are implicit [4,3]) schemes generally impose a second-order-temporal accuracy limitation independent of the accuracy of the LMM chosen as the time differencing approximation. The reason for this will become apparent in Sec. 4. In principle, by altering conventional procedures, $\mathrm{ADI}$ schemes of temporal order greater than 2 can be constructed for PDEs where $A_{0}$-stable LMMs are appropriate; however, the unconditional stabi1ity of the higher order ADI schemes is an open question. The second practical consideration is computer storage. Even if the question of unconditional stability is answered in the affirmative, the scheme must be at least a threestep method since the two-step method (2.6) contains no $A_{0}$-stable third-order subclass.

2.2. One-leg (multistep) methods. A class of methods closely related to LMMs is the one-leg (multistep) methods. The one-leg (k-step) method [5] 
corresponding to $(2.2)$ is

$$
\rho(E) \hat{u}^{n}=\Delta t f\left(\sigma(E) \hat{u}^{n}, \sigma(E) t^{n}\right)
$$

where $\hat{\mathbf{u}}^{\mathrm{n}}$ denotes the one-leg method solution. Formally, the one-leg method (2.17) can be obtained by shifting the operator $\sigma(E)$ inside the argument parenthesis of $f^{n}=f\left(u^{n}, t^{n}\right)$ in the linear multistep formula (2.2). As an example, the trapezoidal formula (2.11) is an LMM and the implicit midpoint rule,

$$
\hat{u}^{n+1}-\hat{u}^{n}=\Delta t f\left(\frac{\hat{u}^{n+1}+\hat{u}^{n}}{2}, t^{n}+\frac{\Delta t}{2}\right),
$$

is the corresponding one-leg method. Dahlquist [5] has proved the following theorem relating solutions of a one-leg method and an LMM: Let $\left\{\hat{u}^{\mathrm{n}}\right\}$ be a vector sequence that satisfies the one-leg difference equation (2.17) and set

$$
\mathrm{u}^{\mathrm{n}}=\sigma(E) \hat{\mathrm{u}}^{\mathrm{n}}
$$

Then $\left\{\mathrm{u}^{\mathrm{n}}\right\}$ satisfies the LMM difference formula (2.2).

It should be noted that LMMs and one-leg methods are equivalent when applied to the linear test equation (2.12) and, consequently, the results of linear stability analysis are the same for both. However, one-leg methods simplify nonlinear stability analysis [5] and, in addition, they are more reliable than LMMs when used with rapidly varying integration step sizes [17]. As applied in this paper, the one-leg formulation makes it easy to construct an ADI scheme for the parabolic Equation (1.1) with time-dependent coefficients.

\subsection{Combined linear multistep methods. If the function $f(u)$ of the} differential equation (2.1) is split into a sum, that is,

$$
\frac{d u}{d t}=f(u, t)=f_{1}(u, t)+f_{2}(u, t) \text {, }
$$


we can construct an integration formula by combining two different LMMs. For examp1e,

$$
\rho(E) u^{n}=\Delta t \sigma_{1}(E) f_{1}^{n}+\Delta t \sigma_{2}(E) f_{2}^{n},
$$

where the subscripts on $\sigma_{1}$ and $\sigma_{2}$ indicate that the coefficients $\beta_{j}$ of the generating polynomial (2.3b) differ for the two functions $f_{1}$ and $f_{2}$. The analogous split one-leg method (2.17) is

$$
\rho(E) \hat{u}^{n}=\Delta t f_{1}\left(\sigma_{1}(E) \hat{u}^{n}, \sigma_{1}(E) t^{n}\right)+\Delta t f_{2}\left(\sigma_{2}(E) \hat{u}^{n}, \sigma_{2}(E) t^{n}\right) .
$$

3. Unconditionally stable schemes. In this section we examine the stability of an implicit method for the parabolic equation (1.1) where the time differencing approximation is an $A_{0}$-stable LMM. Next we modify the scheme by treating the mixed derivative explicitly and determine the criteria for unconditional stability. Although not essential to the final goal of constructing unconditionally stable ADI schemes with the mixed derivative computed explicitly, this intermediate analysis does isolate the stability constraints due to the application of combined LMMs and those due to approximate factorization. Furthermore, the nonfactored scheme is formulated so that the ADI variant follows directly (Sec. 4).

Numerical methods for solving the parabolic equation (1.1) can be obtained by a direct application of the LMM (2.2). Since our interest is in constructing unconditionally stable schemes, we assume that the LMM is $A_{0}$-stable. By comparing (1.1) and (2.1), we identify

$$
f(u)=L u=\left(a \frac{\partial^{2}}{\partial x^{2}}+b \frac{\partial^{2}}{\partial x \partial y}+c \frac{\partial^{2}}{\partial y^{2}}\right) u
$$

where $\mathrm{L}$ is a linear differential operator. For simplicity we assume that the coefficients $a, b, c$ are independent of time. The case of time-dependent 
coefficients is considered in Sec. 6. Insertion of (3.1) into (2.2) yields

$$
\rho(E) u^{n}=\Delta t\left(a \frac{\partial^{2}}{\partial x^{2}}+b \frac{\partial^{2}}{\partial x \partial y}+c \frac{\partial^{2}}{\partial y^{2}}\right) \sigma(E) u^{n} \text {. }
$$

To analyze the stability of (3.2) we assume a solution for the PDE (1.1) (with constant coefficients) of the form

$$
u(x, y, t)=v(t) e^{i\left(k_{1} x+k_{2} y\right)}
$$

where $v(t)$ is the Fourier coefficient and $k_{1}, k_{2}$ are the Fourier variables (wave numbers). Substitution of (3.3) into (1.1) yields an ODE for $v(t)$ :

$$
\frac{d v}{d t}=\lambda v,
$$

where

$$
\lambda=-\left(a k_{1}^{2}+b k_{1} \kappa_{2}+c k_{2}^{2}\right) .
$$

The quadratic form in the parenthesis of $(3.4 \mathrm{~b})$ is positive definite if and only if the inequalities $(1.1 c, d)$ are satisfied. These constraints constitute the parabolicity condition of the PDE and ensure that the solution of (3.4) is damped with time. To complete the stability analysis of the PDE scheme (3.2), we need only consider the stability of the LMMs (2.2) applied to (3.4) with $\lambda<0$. Since we assumed that (2.2) is $A_{0}$-stable, the PDE scheme (3.2) is unconditionally stable. In practice, the spatial derivatives in (3.2) are replaced by appropriate difference quotients; however, as shown at the end of Appendix B, central spatial discretization does not alter the unconditional stability criteria obtained by assuming spatially continuous solutions.

For the one-step methods (2.10), the scheme (3.2) reduces to

$$
u^{n+1}-u^{n}=\Delta t\left(a \frac{\partial^{2}}{\partial x^{2}}+b \frac{\partial^{2}}{\partial x \partial y}+c \frac{\partial^{2}}{\partial y^{2}}\right)\left[\theta u^{n+1}+(1-\theta) u^{n}\right] \text {. }
$$

With central spatial difference approximations, (3.5) is identical to a scheme suggested by Lax and Richtmyer [13]. Their paper appeared about the 
same time the original ADI methods were proposed by Peaceman and Rachford [18] and Douglas [8]. The first ADI methods [8, 18] did not include a mixed derivative term and its presence precludes the construction of an efficient ADI method. A simple way of circumventing this difficulty is to treat the mixed derivative explicitly. One might expect, however, that this would have an adverse effect on the unconditional stability of the algorithm. This stability question is considered in the remainder of this section.

Consider the combined LMM (2.20) where $\sigma_{1}$ and $\sigma_{2}$ are defined as follows. Let (2.2) represent a second-order $A_{0}$-stable LMM and define $(3.6 \mathrm{a}, \mathrm{b}) \quad \sigma_{1}(E)=\sigma(E), \quad \sigma_{2}(E)=\sigma_{\mathrm{e}}(E)$, where $\sigma_{e}(E)$ is a second-order explicit LMM (i.e., $\beta_{k}=0$ in the generating polynomial (2.3b)) with the same generating polynomial $\rho(\zeta)$ as for the A -stable LMM. With these definitions, (2.20) becomes

$$
\rho(E) u^{n}=\Delta t \sigma(E) f_{1}^{n}+\Delta t \sigma_{e}(E) f_{2}^{n}
$$

Henceforth, in reference to a combined implicit-explicit method such as (3.7), we refer to the LMM that defines $\rho(E)$ and $\sigma(E)$ as the generating LMM. The linear stability properties of (3.7) for second-order two-step methods are examined in Appendix A.

For didactic purposes in this section and practical reasons in the following section, we rewrite the LMM (3.7) as

$$
\rho(E) u^{n}-\omega \Delta t \rho(E) f_{1}^{n}=\Delta t[\sigma(E)-\omega \rho(E)] f_{1}^{n}+\Delta t \sigma_{e}(E) f_{2}^{n},
$$

where

$$
\omega=\beta_{k} / \alpha_{k}
$$

The parameter $\omega$ is defined so that the operator $\sigma(E)-\omega \rho(E)$ on the righthand side of (3.8) is at least one degree lower than the operator $\rho(E)$ on the 
left-hand side. This can readily be seen by using the definitions (2.3) and writing out the highest degree term of the operator

$$
\sigma(E)-\omega \rho(E)=\left(\beta_{k-1}-\frac{\beta_{k}}{\alpha_{k}} \alpha_{k-1}\right) E^{k-1}+\ldots .
$$

Consequently, the right-hand side of (3.8) can be computed explicitly, that is, from known data when advancing the numerical solution from $n+k-1$ to $n+k$.

Finally, to apply the combined scheme (3.8) to the PDE (1.1), we split the linear differential operator of (3.1), i.e.,

$$
f_{1}(u)=\left(a \frac{\partial^{2}}{\partial x^{2}}+c \frac{\partial^{2}}{\partial y^{2}}\right) u \text { and } \quad f_{2}(u)=b \frac{\partial^{2} u}{\partial x \partial y} .
$$

By substituting (3.11) into (3.8), we obtain

$$
\begin{aligned}
& {\left[1-\omega \Delta t\left(a \frac{\partial^{2}}{\partial x^{2}}+c \frac{\partial^{2}}{\partial y^{2}}\right)\right] \rho(E) u^{n}} \\
& \quad=\Delta t\left(a \frac{\partial^{2}}{\partial x^{2}}+c \frac{\partial^{2}}{\partial y^{2}}\right)[\sigma(E)-\omega \rho(E)] u^{n}+\Delta t b \frac{\partial^{2}}{\partial x \partial y} \sigma_{e}(E) u^{n} .
\end{aligned}
$$

This formula is implicit for $u_{x x}$ and $u_{y y}$ and explicit for $u_{x y}$.

Remark: The scheme (3.12) with the simplest evaluation of the righthand side has $\sigma_{e}(E)$ given by

$$
\sigma_{e}(E)=[\sigma(E)-\omega \rho(E)] u^{n},
$$

in which case

$$
\operatorname{RHS}(3.12)=\Delta t\left(a \frac{\partial^{2}}{\partial x^{2}}+b \frac{\partial^{2}}{\partial x \partial y}+c \frac{\partial^{2}}{\partial y^{2}}\right)[\sigma(E)-\omega \rho(E)] u^{n} .
$$

Unfortunately, the method with $\sigma_{\mathbf{e}}$ (E) defined by (3.13) is only first-order accurate.

For the stability analysis of (3.12), we consider the second-order, twostep methods where $\rho(E)$ and $\sigma(E)$ are defined by (2.7a) and (2.9) and the 
explicit operator $\sigma_{\mathrm{e}}(E)$ is obtained from (2.9) by setting $\theta=0$,

$$
\sigma_{e}(E)=\left(\xi+\frac{3}{2}\right) E-\left(\xi+\frac{1}{2}\right) \text {. }
$$

The details of the stability analysis are given in Appendix A. Scheme (3.12) is found to be unconditionally stable for all values of $a, b, c$ satisfying equalities $(1.1 c, d)$ if and only if

$$
(3.16 a, b) \quad \xi \leq \theta-1, \quad \xi \geq-\frac{1}{2} \text {. }
$$

The values of the parameters $(\theta, \xi)$ satisfying these inequalities are shown by the shaded region of Fig. 2. Inequality (3.16a) is more restrictive than (2.16a) for the generating second-order, two-step method to be $\mathrm{A}_{\mathrm{o}}$-stable (see Fig. 1). Methods that fall in the region between the lines

$$
\xi=2 \theta-1 \text { and } \xi=\theta-1
$$

and above $\xi=-1 / 2$ are not unconditionally stable for all values of the coefficient $b$ satisfying inequality (1.1d). Note that, with the exception of the two-step trapezoidal formula, none of the methods 1isted in Table 1 falls in the shaded region of Fig. 2.

Remark: It is of interest to note that the explicit operator (3.15) can also be obtained from the implicit operator (2.9) applied to $f^{n}$,

$$
\sigma(E) f^{n}=\theta f^{n+2}+\left(\xi-2 \theta+\frac{3}{2}\right) f^{n+1}-\left(\xi-\theta+\frac{1}{2}\right) f^{n},
$$

by using linear extrapolation, that is,

$$
f^{n+2}=2 f^{n+1}-f^{n}+0\left(\Delta t^{2}\right),
$$

to approximate $\mathrm{f}^{\mathrm{n}+2}$. Although the use of linear extrapolation might sound rather disreputable, the application of an explicit LMM does not.

In the following section we find the rather remarkable result that an approximate factorization of the left-hand side of (3.12) into a product of one-dimensional operators restores most of the parameter space $(\theta, \xi)$ for 
unconditional stability lost by the explicit treatment of the mixed derivative.

4. An ADI scheme: the $\rho(E)$ or $\Lambda$ formulation. In the procedure suggested in [20] for designing ADI schemes, the implicit operator to be inverted is constructed so that the unknown variable to be determined at each time step is $\rho(E) u^{n}$. In the absence of a mixed derivative this choice ensures that approximate factorization into a product of one-dimensional operators does not upset either the temporal accuracy (second-order) or the unconditional stability of the scheme. In this section we construct an ADI scheme in the $\rho(E)$ formulation with the mixed derivative treated explicitly with secondorder accuracy and examine the stability of the resulting algorithm.

If the spatial derivatives appearing in (3.12) are replaced by appropriate difference quotients, then one obtains in general an enormous linear system to solve for $\rho(E) u^{n}$. This difficulty can be overcome by an approximate factorization of the left-hand side of (3.12) which reduces the problem to a product of one-dimensional spatial operators; that is,

$$
\text { (4.I) } \begin{aligned}
\left(1-\omega \Delta \operatorname{ta} \frac{\partial^{2}}{\partial x^{2}}\right) & \left(1-\omega \Delta t c \frac{\partial^{2}}{\partial y^{2}}\right) \rho(E) u^{n} \\
& =\Delta t\left(a \frac{\partial^{2}}{\partial x^{2}}+c \frac{\partial^{2}}{\partial y^{2}}\right)[\sigma(E)-\omega \rho(E)] u^{n}+\Delta t b \frac{\partial^{2}}{\partial x \partial y} \sigma_{e}(E) u^{n} .
\end{aligned}
$$

Comparison of the left-hand sides of (4.1) and (3.12) shows that they differ by the cross-product term

$$
\omega^{2} \Delta t^{2} a \frac{\partial^{2}}{\partial x^{2}} c \frac{\partial^{2}}{\partial y^{2}} \rho(E) u^{n} .
$$

But by expanding $\rho(E) u^{n}$ in a Taylor series about $u^{n}$ and using the consistency and normalization conditions $(2.5 a, b)$, one obtains 


$$
\rho(E) u^{n}=\Delta t \frac{\partial u^{n}}{\partial t}+0\left(\Delta t^{\alpha+1}\right), \quad \alpha \geq 1
$$

Consequent1y, the cross-product term (4.2)

$$
\omega^{2} \Delta t^{2} a \frac{\partial^{2}}{\partial x^{2}} c \frac{\partial^{2}}{\partial y^{2}} \rho(E) u^{n}=\omega^{2} \Delta t^{3} a \frac{\partial^{2}}{\partial x^{2}} c \frac{\partial^{2}}{\partial y^{2}} \frac{\partial}{\partial t} u^{n}+O\left(\Delta t^{4}\right)
$$

$$
=0\left(\Delta t^{3}\right)
$$

is a third-order term and the formal accuracy of the scheme (3.12) is not upset by the approximate factorization (4.1).

In practice, the second-order, two-step methods defined by (2.7a) and (2.9) are of primary interest and in the remainder of this section we limit our attention to these methods. The explicit operator $\sigma_{e}(E)$ is given by (3.15). In numerical algorithms for partial differential equations it is conventional to use $\mathrm{n}+1$ as the most advanced time level; hence, we multiply (4.1) by $\mathrm{E}^{-1}$. The shifted difference operators for the second-order, twostep method can be written as

$$
\begin{array}{rlrl}
(4.5 a) & \Lambda u^{n}=E^{-1} \rho(E) u^{n} & = & {\left[(1+\xi) E-(1+2 \xi)+\xi E^{-1}\right] u^{n},} \\
(4.5 b) & & =[(1+\xi) \Delta-\xi \nabla] u^{n}, \\
(4.5 c) & & =(1+\xi) u^{n+1}-(1+2 \xi) u^{n}+\xi u^{n-1}, \\
(4.6 a) & E^{-1} \sigma(E) u^{n} & =\left[\theta E+\left(\xi-2 \theta+\frac{3}{2}\right)-\left(\xi-\theta+\frac{1}{2}\right) E^{-1}\right] u^{n}, \\
(4.6 b) & =\left[1+\theta \Delta+\left(\xi-\theta+\frac{1}{2}\right) \nabla\right] u^{n}, \\
\text { (4.7a) } & E^{-1} \sigma_{e}(E) u^{n} & =\left[\left(\xi+\frac{3}{2}\right)-\left(\xi+\frac{1}{2}\right) E^{-1}\right] u^{n}, \\
\text { (4.7b }) & & &
\end{array}
$$

where the symbols $\Delta$ and $\nabla$ are classical forward and backward difference operators defined by 


$$
\Delta u^{n}=u^{n+1}-u^{n}, \quad \nabla u^{n}=u^{n}-u^{n-1} .
$$

As a notational convenience, we have denoted the operator $E^{-1} \rho(E)$ by $\Lambda$. From (4.5) and (4.6) there follows

$$
\begin{aligned}
E^{-1}[\sigma(E)-\omega \rho(E)] u^{n} & =\left[\left(\xi-\omega+\frac{3}{2}\right)-\left(\xi-\omega+\frac{1}{2}\right) E^{-1}\right] u^{n} \\
& =\left[1+\left(\xi-\omega+\frac{1}{2}\right) \nabla\right] u^{n},
\end{aligned}
$$

where

$$
\omega=\frac{\theta}{1+\xi}
$$

The factored scheme (4.1) becomes

$$
\begin{aligned}
& \left(1-\omega \Delta \operatorname{ta} \frac{\partial^{2}}{\partial x^{2}}\right)\left(1-\omega \Delta t c \frac{\partial^{2}}{\partial y^{2}}\right) \Lambda u^{n} \\
& =\Delta t\left(a \frac{\partial^{2}}{\partial x^{2}}+c \frac{\partial^{2}}{\partial y^{2}}\right)\left[1+\left(\xi-\omega+\frac{1}{2}\right) \nabla\right] u^{n}+\Delta t b \frac{\partial^{2}}{\partial x \partial y}\left[1+\left(\xi+\frac{1}{2}\right) \nabla\right] u^{n} .
\end{aligned}
$$

The computational sequence to implement the factored scheme (4.11) as an ADI method is not unique, but an obvious choice is

$$
\left(1-\omega \Delta \text { ta } \frac{\partial^{2}}{\partial x^{2}}\right) \Lambda u^{*}=\text { RHS (4.11), }
$$

$$
\left(1-\omega \Delta t c \frac{\partial^{2}}{\partial y^{2}}\right) \Lambda u^{n}=\Lambda u^{*}
$$

$$
(1+\xi) u^{n+1}=\Lambda u^{n}+(1+2 \xi) u^{n}-\xi u^{n-1},
$$

where $\Lambda u^{*}$ is a dummy temporal difference.

Remark: If the first-order explicit method with $\sigma_{e}(E)$ defined by (3.13) were used rather than a second-order method, the right-hand side of (4.1) would be the same as (3.14). In this case, the right-hand side of (4.11) would be replaced by

$$
\Delta t\left(a \frac{\partial^{2}}{\partial x^{2}}+b \frac{\partial^{2}}{\partial x \partial y}+c \frac{\partial^{2}}{\partial y^{2}}\right)\left[1+\left(\xi-\omega+\frac{1}{2}\right) \nabla\right] u^{n}
$$


where we have used (4.9). Although the resulting scheme is first-order accurate in time for the mixed derivative, it is unconditionally stable for values of $(\theta, \xi)$ in the shaded region of Fig. 1. This result was established in [20, Appendix A] by constructing the scheme so that the characteristic equation (which determines the stability of the method) for the factored partial difference equation had the same form as the characteristic equation (2.13) for the ordinary differential equation.

The scheme (4.11) is second-order accurate in the mixed derivative but the characteristic equation no longer has the form (2.13). Consequently, the stability analysis must be redone in a less elegant manner than in [20] and is carried out in Appendix B. The stability analysis of Appendix B is for the general two-step ADI scheme developed in the following section. The $\Lambda$ formulation (4.11) is a special case of the general two-step scheme and is found to be unconditionally stable for all values of $a, b, c$ satisfying inequalities $(1.1 c, d)$ if and only if
$(4.14 a, b)$
$\theta \geq \frac{2(1+\xi)^{2}}{3+4 \xi}$
$\xi \geq-\frac{1}{2}$

The parameter space $(\theta, \xi)$ satisfying these inequalities is shown by the shaded region of Fig. 3. The inequality $(4.14 a)$ is more stringent than (2.16a), and methods that fall in the region between the curves

$$
\text { (4.15) } \xi=2 \theta-1 \text { and } \theta=\frac{2(1+\xi)^{2}}{3+4 \xi}
$$

and above $\xi=-1 / 2$ are not unconditionally stable for all values of the coefficient b satisfying inequality (1.1d). This includes such popular schemes as the trapezoidal formula and Lees method (see, e.g., [2]). However, there remains a large class of unconditionally stable methods including, e.g., the backward differentiation formula (see Table 1). 
5. A general two-step ADI scheme. In the ADI formulation described in the previous section it is essential that the unknown variable be at least a first difference to ensure that the approximate factorization does not degrade the second-order accuracy of the scheme; for example, the unknown variable $E^{-1} \rho(E) u^{n}=\Lambda u^{n}$ is an approximation to $\Delta t(\partial u / \partial t)$ (see Eq. (4.3)). The choice of unknown variable is not unique and in fact the most general form of a first difference using data from the three levels $n-1, n, n+1$ can be written as

$$
\begin{aligned}
u^{n+1}-(1+\alpha) u^{n}+\alpha u^{n-1} & =(\Delta-\alpha \nabla) u^{n} \\
& =(1-\alpha) \Delta t \frac{\partial u^{n}}{\partial t}+(1+\alpha) \frac{\Delta t^{2}}{2} \frac{\partial^{2} u^{n}}{\partial t^{2}}+0\left(\Delta t^{3}\right),
\end{aligned}
$$

where $\Delta$ and $\nabla$ are the forward and backward operators defined by (4.8) and $\alpha$ is an arbitrary real constant. The undivided difference (5.1) is a second difference if $\alpha=1$ and a first difference otherwise. The most accurate first difference is for $\alpha=-1$.

\subsection{General formulation. A general formulation of two-step ADI schemes} is obtained if we choose $(\Delta-a \nabla) u^{n}$ as the unknown variable. We first rewrite the LMM (3.7) in a convenient form for the construction of an ADI scheme with $(\Delta-\alpha \nabla) \mathrm{u}^{\mathrm{n}}$ as the unknown variable. Multiplying (3.7) by $\mathrm{E}^{-1}$ and inserting the operators $E^{-1} \rho(E), E^{-1} \sigma(E)$, and $E^{-1} \sigma_{e}(E)$ defined by $(4.5 b),(4.6 b)$, and $(4.7 b)$, one can rewrite the resulting two-step method as

$$
\begin{aligned}
\Delta u^{n}-\omega \Delta t \Delta f_{1}^{n}= & \frac{\Delta t}{1+\xi}\left[1+\left(\xi-\theta+\frac{1}{2}\right) \nabla\right]\left(f_{1}^{n}+f_{2}^{n}\right) \\
& +\omega \Delta t \nabla f_{2}^{n}+\frac{\xi}{1+\xi} \nabla u^{n}
\end{aligned}
$$

After subtracting $\alpha \nabla u^{n}-\alpha \omega \Delta t \nabla f_{l}^{n}$ from both sides, there follows 
(5.3) $(\Delta-\alpha \nabla) u^{n}-\omega \Delta t(\Delta-\alpha \nabla) f_{1}^{n}=\frac{\Delta t}{1+\xi}\left[1+\left(\xi-\theta+\frac{1}{2}\right) \nabla\right]\left(f_{1}^{n}+f_{2}^{n}\right)$

$$
+\omega \Delta t \nabla f_{2}^{n}+\alpha \omega \Delta t \nabla f_{1}^{n}+\left(\frac{\xi}{1+\xi}-\alpha\right) \nabla u^{n} \text {. }
$$

Using (3.11), one obtains

$$
\begin{aligned}
& {\left[1-\omega \Delta t\left(a \frac{\partial^{2}}{\partial x^{2}}+c \frac{\partial^{2}}{\partial y^{2}}\right)\right](\Delta-\alpha \nabla) u^{n}} \\
& =\frac{\Delta t}{1+\xi}\left(a \frac{\partial^{2}}{\partial x^{2}}+b \frac{\partial^{2}}{\partial x \partial y}+c \frac{\partial^{2}}{\partial y^{2}}\right)\left[1+\left(\xi-\theta+\frac{1}{2}\right) \nabla\right] u^{n} \\
& \quad+\omega \Delta t b \frac{\partial^{2}}{\partial x \partial y} \nabla u^{n}+\alpha \omega \Delta t\left(a \frac{\partial^{2}}{\partial x^{2}}+c \frac{\partial^{2}}{\partial y^{2}}\right) \nabla u^{n}+\left(\frac{\xi}{1+\xi}-\alpha\right) \nabla u^{n}
\end{aligned}
$$

The spatially factored form of $(5.4)$ is

$$
\left(1-\omega \Delta \mathrm{ta} \frac{\partial^{2}}{\partial x^{2}}\right)\left(1-\omega \Delta \mathrm{tc} \frac{\partial^{2}}{\partial y^{2}}\right)(\Delta-\alpha \nabla) \mathrm{u}^{\mathrm{n}}=\operatorname{RHS}(5.4),
$$

which can be implemented as

$$
\left(1-\omega \Delta \operatorname{ta} \frac{\partial^{2}}{\partial x^{2}}\right)(\Delta-\alpha \nabla) u^{*}=\operatorname{RHS}(5.4),
$$

$$
\left(1-\omega \Delta t c \frac{\partial^{2}}{\partial y^{2}}\right)(\Delta-\alpha \nabla) u^{n}=(\Delta-\alpha \nabla) u^{*},
$$

$$
u^{n+1}=(\Delta-\alpha \nabla) u^{n}+(1+\alpha) u^{n}-\alpha u^{n-1} .
$$

The stability analysis for the general factored scheme (5.5) is given in Appendix B. The scheme is found to be unconditionally stable for all values of $a, b, c$ satisfying inequalities $(1 c, d)$ if and only if

$$
(5.7 \mathrm{a}, \mathrm{b}, \mathrm{c}) \theta \geq \frac{2(1+\xi)}{2+\sqrt{\frac{(1+\alpha)(1+2 \xi)}{1+\xi}}}, \quad \xi \geq-\frac{1}{2}, \quad-1 \leq \alpha \leq 1 \text {. }
$$

The parameter space $(\theta, \xi)$ satisfying these inequalities is indicated in Fig. 4 for several values of $\alpha$. For a given value of $\alpha$, the stable range is to the right of the curve labeled with that value of $\alpha$ and above the curve $\xi=-1 / 2$. The extent of the $(\theta, \xi)$ parameter space for unconditional 
stability is a monotone increasing function of $\alpha$ in the range [-1,1] with the smallest region for $\alpha=-1$ and the largest for $\alpha=+1$. Inequality (5.7a) is more stringent than (2.16a) as is apparent in Fig. 4. Along the line $\xi=0$, inequality $(5.7 a)$ becomes

$$
\theta \geq \frac{2}{2+\sqrt{1+\alpha}}, \quad(\xi=0)
$$

and the smallest allowed value for $\theta$ occurs when $\alpha=1$, in which case (5.8) becomes

$$
\theta \geq \frac{1}{1+\sqrt{1 / 2}} \simeq 0.586, \quad(\xi=0)
$$

Along the lower stability boundary $\xi=-1 / 2$, inequality (5.7a) becomes

$$
\theta \geq \frac{1}{2}, \quad\left(\xi=-\frac{1}{2}\right)
$$

which is independent of the parameter $\alpha$. As a consequence of inequalities (5.9) and (5.10), such popular implicit methods as the trapezoidal formula $(\theta=1 / 2, \xi=0)$ and Lees method $(\theta=1 / 3, \xi=-1 / 2)$ are not unconditionally stable for all values of the coefficient $b$ satisfying inequality $(1.1 \mathrm{~d})$.

If the parameter $\alpha$ is chosen to be $\xi /(1+\xi)$, scheme (5.5) reduces to the $\Lambda$ formulation of Sec. 4. This ADI method has the peculiar property that the unknown variable depends on the parameter $\xi$, that is, on the particular LMM chosen. The parameter space $(\theta, \xi)$ for which the $\Lambda$ formulation is unconditionally stable is shown by the shaded region of Fig. 2 and the extent of the region is nearly as large as that for (5.5) with $\alpha=1$.

\subsection{Special cases of general formulation. Various constant values of} $\alpha$ in the range $[-1,1]$ produce useful and interesting algorithms and we consider several in greater detail. The schemes are named according to the 
classical difference operator represented by (5.1) for the chosen values of $\alpha$.

5.2a. The $\Delta$ formulation $(\alpha=0)$. If $\alpha$ is chosen to be zero, the general scheme $(5.5)$ reduces to

$$
\begin{aligned}
\left(1-\omega \Delta t a \frac{\partial^{2}}{\partial x^{2}}\right)(1- & \left.\omega \Delta t c \frac{\partial^{2}}{\partial y^{2}}\right) \Delta u^{n} \\
= & \frac{\Delta t}{1+\xi}\left(a \frac{\partial^{2}}{\partial x^{2}}+b \frac{\partial^{2}}{\partial x \partial y}+c \frac{\partial^{2}}{\partial y^{2}}\right)\left[1+\left(\xi-\theta+\frac{1}{2}\right) \nabla\right] u^{n} \\
& +\omega \Delta t b \frac{\partial^{2}}{\partial x \partial y} \nabla u^{n}+\frac{\xi}{1+\xi} \nabla u^{n}
\end{aligned}
$$

which we call the $\Delta$ formulation $[1,19]$ since $\Delta \mathrm{u}^{\mathrm{n}}$ is the unknown variable. The parameter space for unconditional stability is given by the inequalities (5.7a,b) with $\alpha=0$ and is shown graphically in Fig. 4.

5.2b. The $\delta^{2}$ formulation $(\alpha=1)$. In the general ADI formulation (5.5) the unknown variable $(\Delta-\alpha \nabla) \mathrm{u}^{\mathrm{n}}$ is an approximation to $\Delta \mathrm{t}(\partial \mathrm{u} / \partial \mathrm{t})$ if $\alpha \neq 1$ (see Eq. (5.1)). A less natural choice for the unknown variable for a firstorder (temporal) differential equation is the second difference obtained when $\alpha=1$. In this case, (5.1) becomes

$$
(\Delta-\nabla) u^{n}=\delta^{2} u^{n}=\Delta t^{2} \frac{\partial^{2} u^{n}}{\partial t^{2}}+0\left(\Delta t^{4}\right),
$$

where the classical second difference operator $\delta^{2}$ is defined by

$$
\delta^{2} u^{n}=u^{n+1}-2 u^{n}+u^{n-1}
$$

The possibility of using $\delta^{2} u^{n}$ as the unknown variable does not arise for linear one-step methods (e.g., the trapezoidal formula (2.11)) since these methods only involve two time levels. 
If $\alpha$ is set equal to one in the general two-step scheme (5.5), we obtain

$$
\text { (5.14) } \begin{aligned}
(1-\omega \Delta t a & \left.\frac{\partial^{2}}{\partial x^{2}}\right)\left(1-\omega \Delta t c \frac{\partial^{2}}{\partial y^{2}}\right) \delta^{2} u^{n} \\
& =\frac{\Delta t}{1+\xi}\left(a \frac{\partial^{2}}{\partial x^{2}}+b \frac{\partial^{2}}{\partial x \partial y}+c \frac{\partial^{2}}{\partial y^{2}}\right)\left[1+\left(\xi+\frac{1}{2}\right) \nabla\right] u^{n}-\frac{1}{1+\xi} \nabla u^{n} .
\end{aligned}
$$

A possible advantage of the $\delta^{2}$ formulation is that the cross-term error introduced by the approximate factorization is one order higher than in the general $(\Delta-\alpha \nabla)$ formulation with $\alpha \neq 1$. By comparing the left-hand sides of (5.4) and (5.5), we find they differ by

$$
\omega^{2} \Delta t^{2} a \frac{\partial^{2}}{\partial x^{2}} c \frac{\partial^{2}}{\partial y^{2}}(\Delta-\alpha \nabla) u^{n}
$$

which, for $\alpha=1$, becomes

$$
\begin{aligned}
\omega^{2} \Delta t^{2} a \frac{\partial^{2}}{\partial x^{2}} c \frac{\partial^{2}}{\partial y^{2}} \delta^{2} u^{n} & =\omega^{2} \Delta t^{4} a \frac{\partial^{2}}{\partial x^{2}} c \frac{\partial^{2}}{\partial y^{2}} \frac{\partial^{2} u^{n}}{\partial t^{2}}+0\left(\Delta t^{5}\right), \\
& =0\left(\Delta t^{4}\right),
\end{aligned}
$$

where we have used (5.12). The cross-term error for the $\Lambda=E^{-1} \rho(E)$ formulation is given by (4.4). The parameter space for which the $\delta^{2}$ formulation is unconditionally stable is given by inequalities $(5.7 \mathrm{a}, \mathrm{b})$ with $\alpha=1$ and is shown graphically in Fig. 4.

A distinct disadvantage of the $\delta^{2}$ formulation occurs when it is applied to convective (hyperbolic) model equations as briefly discussed in section 8 .

5.2c. The $2 \mu \delta$ formulation $(\alpha=-1)$. As a final special case of the generalized formulation we choose $\alpha=-1$, that is,

$$
\Delta-\alpha \nabla=\Delta+\nabla=2 \mu \delta,
$$

where $2 \mu \delta$ is the classical central difference operator

$$
2 \mu \delta u^{n}=u^{n+1}-u^{n-1} \text {. }
$$


The scheme (5.5) becomes

$$
\text { (5.18) } \begin{aligned}
\left(1-\omega \Delta t a \frac{\partial^{2}}{\partial x^{2}}\right)(1 & \left.-\omega \Delta t c \frac{\partial^{2}}{\partial y^{2}}\right) 2 \mu \delta u^{n} \\
= & \frac{\Delta t}{1+\xi}\left(a \frac{\partial^{2}}{\partial x^{2}}+b \frac{\partial^{2}}{\partial x \partial y}+c \frac{\partial^{2}}{\partial y^{2}}\right)\left[1+\left(\xi-2 \theta+\frac{1}{2}\right) \nabla\right] u^{n} \\
& +2 \omega \Delta t b \frac{\partial^{2}}{\partial x \partial y} \nabla u^{n}+\frac{1+2 \xi}{1+\xi} \nabla u^{n}
\end{aligned}
$$

The parameter space for unconditional stability of this formulation is given by inequalities $(5.7 \mathrm{a}, \mathrm{b})$ with $\alpha=-1$,

$$
\theta \geq 1+\xi, \quad \xi \geq-\frac{1}{2},
$$

which is identical to the stability space for the unfactored scheme (3.12) (see inequality (3.16) and Fig. 2).

5.3. Algorithm selection. From the class of unconditionally stable methods one can choose a scheme with properties that are desirable with regard to computer storage, computational simplicity, and temporal behavior when applied to stiff problems and/or problems with nonsmooth data. The choice generally requires a compromise.

Consider, for example, the $\Lambda$ formulation (4.11) of Sec. 4. The computation of the right-hand side of (4.11) is obviously simplified if we set

$$
\xi-\omega+\frac{1}{2}=0 \text {. }
$$

Since $\omega=\theta /(1+\xi)$, this equation can be rewritten as

$$
\theta=(\xi+1)\left(\xi+\frac{1}{2}\right)
$$

it is plotted in Fig. 3. Another variant is obtained by rewriting the righthand side of (4.11) as 


$$
\begin{aligned}
\operatorname{RHS}(4.11)= & \Delta t\left(a \frac{\partial^{2}}{\partial x^{2}}+c \frac{\partial^{2}}{\partial y^{2}}\right)\left[\left(\xi-\omega+\frac{3}{2}\right)-\left(\xi-\omega+\frac{1}{2}\right) E^{-1}\right] u^{n} \\
& +\Delta t b \frac{\partial^{2}}{\partial x \partial y}\left[1+\left(\xi+\frac{1}{2}\right) \nabla\right] u^{n},
\end{aligned}
$$

where we have used (4.9a). The calculation of (5.22) is simplified if we let

$$
\xi-\omega+\frac{3}{2}=0
$$

which can be rewritten as

$$
\theta=(\xi+1)\left(\xi+\frac{3}{2}\right)
$$

it is also plotted in Fig. 3. If in addition, we choose $\xi=-1 / 2$, then (5.22) becomes simply

$$
\operatorname{RHS}(4.11)=\Delta t\left(a \frac{\partial^{2}}{\partial x^{2}}+c \frac{\partial^{2}}{\partial y^{2}}\right) u^{n-1}+\Delta t b \frac{\partial^{2} u^{n}}{\partial x \partial y} .
$$

In this special case, each spatial derivative on the right-hand side of (4.11) requires evaluation at only a single time level. The time differencing $(\theta=1 / 2, \xi=-1 / 2)$ corresponds to the two-step trapezoidal formula (see Table 1). $A_{0}-$ and A-stable methods along the bottom boundary $\xi=-1 / 2$ of Fig. 1 are "symmetric" schemes. These methods have the unfortunate property that the modulus of at least one root of the characteristic equation (2.13) approaches 1 as $\lambda \Delta t \rightarrow \infty$. Consequently, these methods can produce slowly decaying numerical oscillations when applied to stiff problems. This observation illustrates that computational simplicity should not provide the sole basis for selecting a time-differencing scheme.

The computation of the right-hand side of the $\Delta$ formulation (5.11) is obviously simplified if we set

$$
\xi-\theta+\frac{1}{2}=0
$$

This special case of the $\Delta$ formulation was given by the authors in [1; see 
Eq. (4.2)]. The $\Delta$ formulation is particularly attractive for its simplicity in programming logic and minimal computing storage requirements. Finally, the $\delta^{2} u$ formulation (5.14) has the computational advantage that all spatial derivatives on the right-hand side operate on the same function, that is, $[1+(\xi+1 / 2) \nabla] \mathrm{u}^{\mathrm{n}}$. For the special case $\xi=-1 / 2$, the function is conveniently $u^{n}$.

A1though considerations of computer storage and computational simplicity may not be particularly important for the simple model equation (1.1), they are of primary concern when one deals with more complicated parabolic equations such as the compressible Navier-Stokes equations (see, e.g., [1,21]).

\subsection{General formulation with no mixed derivative. It is important to} note that if $b \equiv 0$, that is, there is no mixed derivative, then inequalities (5.7) are replaced by

$$
\xi \leq 2 \theta-1, \quad \xi \geq-\frac{1}{2}, \quad-1 \leq \alpha \leq 1,
$$

and the general two-step ADI formula (5.5) is unconditionally stable for the same values of $(\theta, \xi)$ as for the original second-order, two-step method (see inequalities (2.16) and Fig. 1). In the absence of mixed derivatives, the natural extension of (5.5) to three spatial dimensions is also unconditionally stable for values of $(\alpha, \theta, \xi)$ satisfying inequalities (5.27).

It is appropriate to mention the relation between the Douglas-Gunn method [9, Sec. 3] for multilevel difference schemes and the general two-step ADI scheme (5.6) in the absence of a mixed derivative, that is, $b=0$. The difference (5.1) corresponds to the difference

$$
u^{n+1}-u_{*}^{n+1}
$$

in the Douglas-Gunn development where 
$(5.28 b)$

$$
u_{*}^{n+1}=\phi_{0} u^{n}+\phi_{1} u^{n-1}, \quad \phi_{0}+\phi_{1}=1
$$

Hence, on comparing (5.28) and (5.1), one finds that

$$
\phi_{0}=1+\alpha, \quad \phi_{1}=-\alpha .
$$

Douglas and Gunn give a formal procedure for devising an ADI scheme from a fully implicit scheme supplied by the user. For example, consider the secondorder, two-step method ((3.2) with $b=0)$, where $\rho(E)$ and $\sigma(E)$ are defined by $(2.7 a)$ and $(2.9)$ and $(\theta, \xi)$ satisfy inequalities $(2.16)$. If we apply the formulas (3.7) of [9], we obtain an $A D I$ algorithm that can be shown to be equivalent to $(5.6)$. The resulting scheme is unconditionally stable for $-1 \leq \alpha \leq 1$ since the LMM is Ao-stable. Recall that the discussion of this paragraph is only for the case of no mixed derivative.

6. Time-dependent coefficients. If the coefficients $a, b, c$ of the PDE (1.1) are functions of time, a difficulty arises when we insert (3.11) into (3.8) since

$$
\rho(E)\left[a\left(t^{n}\right) \frac{\partial^{2} u^{n}}{\partial x^{2}}+c\left(t^{n}\right) \frac{\partial^{2} u^{n}}{\partial y^{2}}\right] \neq\left[a\left(t^{n}\right) \frac{\partial^{2}}{\partial x^{2}}+c\left(t^{n}\right) \frac{\partial^{2}}{\partial y^{2}}\right] \rho(E) u^{n} .
$$

This is not an equality because the time dependence of the coefficients cannot be neglected when the temporal-difference operator $\rho(E)$ is applied. This problem can be avoided if we begin with the one-leg method (2.21) instead of the conventional LMM.

With $\sigma_{1}$ and $\sigma_{2}$ defined by (3.6), the one-leg method (2.21) is

$$
\rho(E) \hat{u}^{n}=\Delta t f_{1}\left(\sigma(E) \hat{u}^{n}, \sigma(E) t^{n}\right)+\Delta t f_{2}\left(\sigma_{e}(E) \hat{u}^{n}, \sigma_{e}(E) t^{n}\right) .
$$

For the PDE (1.1) we identify $f_{1}$ and $f_{2}$ as (3.11) and obtain 


$$
\begin{aligned}
\rho(E) \hat{\mathrm{u}}^{\mathrm{n}}= & \Delta t\left[a(\overline{\mathrm{t}}) \frac{\partial^{2}}{\partial x^{2}}+c(\overline{\mathrm{t}}) \frac{\partial^{2}}{\partial \mathrm{y}^{2}}\right] \sigma(E) \hat{\mathrm{u}}^{\mathrm{n}} \\
& +\Delta t\left[b\left(\overline{\mathrm{t}}_{e}\right) \frac{\partial^{2}}{\partial x \partial y}\right] \sigma_{e}(E) \hat{\mathrm{u}}^{\mathrm{n}},
\end{aligned}
$$

where $\bar{t}$ and $\bar{t}_{e}$ are defined by

$$
(6.4 a, b) \quad \bar{t}=\sigma(E) t^{n}, \quad \bar{t}_{e}=\sigma_{e}(E) t^{n} \text {. }
$$

If (6.3) is modified to the prefactored form by subtracting

$$
\omega \Delta t\left[a(\bar{t}) \frac{\partial^{2}}{\partial x^{2}}+c(\bar{t}) \frac{\partial^{2}}{\partial y^{2}}\right] \rho(E) \hat{u}^{n}
$$

from each side, we obtain

$$
\text { (6.5) } \begin{aligned}
&\left\{1-\omega \Delta t\left[a(\bar{t}) \frac{\partial^{2}}{\partial x^{2}}+c(\bar{t}) \frac{\partial^{2}}{\partial y^{2}}\right]\right\} \rho(E) \hat{u}^{n} \\
&= \Delta t\left[a(\bar{t}) \frac{\dot{o}^{2}}{\partial x^{2}}+c(\bar{t}) \frac{\partial^{2}}{\partial y^{2}}\right][\sigma(E)-\omega \rho(E)] \hat{u}^{n} \\
&+\Delta t b\left(\bar{t}_{e}\right) \frac{\partial^{2}}{\partial x \partial y} \sigma_{e}(E) \hat{u}^{n} .
\end{aligned}
$$

The prefactored form (6.5) is identical to (3.12) where a, c, and b are evaluated at $\bar{t}$ and $\bar{t}_{e}$ defined by (6.4). Consequently, the factored scheme (4.1) is valid for time-dependent coefficients provided a, c,b are evaluated at the appropriate times $\overline{\mathrm{t}}$ and $\overline{\mathrm{t}}_{\mathrm{e}}$.

For second-order, two-step methods, the shifted-difference operators are defined by (4.6) and (4.7). For this case

$$
\begin{aligned}
& E^{-1} \bar{t}=E^{-1} \sigma(E) t^{n}=t^{n}+\left(\xi+\frac{1}{2}\right) \Delta t, \\
& E^{-1} \bar{t}_{e}=E^{-1} \sigma_{e}(E) t^{n}=t^{n}+\left(\xi+\frac{1}{2}\right) \Delta t,
\end{aligned}
$$

and hence the time-dependent coefficients $a, b, c$ are all evaluated at the same time which we denote by 


$$
t^{*}=t^{n}+\left(\xi+\frac{1}{2}\right) \Delta t
$$

Therefore, the ADI scheme (4.12) is valid for time-dependent coefficients evaluated at $t^{*}$. Likewise, the same statement applies to the general twostep ADI scheme (5.6).

7. Numerical examples. In this section the ADI methods of the previous sections are used to solve the parabolic equation (1.1) for a test problem with variable coefficients. The purpose of these numerical experiments is not to find the optimum scheme but to demonstrate by numerical example that each of the formulations $-\Lambda, \Delta$, and $\delta^{2}-$ achieves the purported second-order accuracy. In addition, we demonstrate the detrimental effect on the accuracy if the mixed derivative is treated with a first-order-accurate method or the variable coefficients are not evaluated at the proper time level.

For the example problem, the coefficients are

$$
\begin{aligned}
& a(x, y, t)=\left(\frac{1}{2} x^{2}+y^{2}\right)\left(1+t^{2}\right), \\
& b(x, y, t)=-\left(x^{2}+y^{2}\right)\left(1+t^{2}\right), \\
& c(x, y, t)=\left(x^{2}+\frac{1}{2} y^{2}\right)\left(1+t^{2}\right) .
\end{aligned}
$$

An exact solution is

$$
u(x, y, t)=\left(x^{2} y+x y^{2}\right) \exp \left[-\left(1+\frac{t^{2}}{3}\right) t\right]
$$

Numerical solutions were computed on the unit square $(0 \leq x, y \leq 1)$ with the initial and boundary values computed from (7.2). For example, the initial condition is

$$
\mathrm{u}(\mathrm{x}, \mathrm{y}, 0)=\mathrm{x}^{2} \mathrm{y}+\mathrm{xy}^{2}, \quad 0 \leq \mathrm{x}, \mathrm{y} \leq 1 \text {. }
$$

This model problem is a variant of an example given by McKee and Mitchel1 [15] modified so that the coefficients $a, b, c$ are time-dependent. 
In the numerical computations of this section, the spatial derivatives were approximated by the following central difference approximations

$$
\left.\frac{\partial^{2} Q}{\partial x^{2}}\right|_{j, k}=\frac{\delta^{2} Q_{j, k}}{\Delta x^{2}}+0\left(\Delta x^{2}\right),
$$

$$
\left.\frac{\partial^{2} Q}{\partial y^{2}}\right|_{j, k}=\frac{\delta_{y}^{2} Q_{j, k}}{\Delta y^{2}}+0\left(\Delta y^{2}\right),
$$

$$
\left.\frac{\partial Q}{\partial x \partial y}\right|_{j, k}=\frac{(\mu \delta)_{x}(\mu \delta) y}{\Delta x \Delta y} Q_{j, k}+0\left(\Delta x^{2}, \Delta y^{2}\right)
$$

$$
=\frac{1}{4 \Delta x \Delta y}\left(Q_{j+1, k+1}-Q_{j+1, k-1}-Q_{j-1, k+1}+Q_{j-1, k-1}\right),
$$

where $x=j \Delta x$ and $y=k \Delta y$. Here $\delta$ and $\mu$ are classical finite-difference operators defined by

$$
\delta_{x} Q_{j}=Q_{j+1 / 2}-Q_{j-1 / 2}, \quad 2 \mu_{x} Q_{j}=Q_{j+1 / 2}+Q_{j-1 / 2},
$$

and hence

$$
\begin{aligned}
\delta_{x}^{2} Q_{j} & =Q_{j+1}-2 Q_{j}+Q_{j-1}, \\
2(\mu \delta)_{x} Q_{j} & =Q_{j+1}-Q_{j-1}, \text { etc. }
\end{aligned}
$$

Consider, for example, the $\Lambda$ formulation (4.12). With the spatial derivatives replaced by the central-difference quotients (7.4)-(7.6), the $x$ - and $y$-operators on the left-hand side of $(4.12 a, b)$ each requires the solution of a tridiagonal system. There is a well-known and highly efficient solution algorithm for tridiagonal systems (see, e.g., [11, p. 55]). The solution of the $\mathrm{x}$-operator (4.12a) (along each y-constant 1ine) requires the dummy temporal difference $\Lambda \mathrm{u}^{*}$ along the left and right boundaries. In problems considered in this section, we assume that $u(t)$ is given on the boundaries, and consequently $\Lambda u^{*}$ can be determined by an explicit calculation using (4.12b) applied along both the left- and right-hand boundaries. 
This is the initial calculation made when advancing the solution from $n$ to $\mathrm{n}+1$. Application of the general two-step ADI scheme (5.6) requires an analogous computation of a dummy temporal difference along the left and right boundaries.

Since the algorithms considered in this paper are, in general, two-step (temporal) schemes, a solution at $t=\Delta t$ is needed together with the initial condition to start the computation. For the numerical examples computed herein, the exact solution (7.2) at $t=\Delta t$ was used to provide the additional level of data. In practice, one can use (4.12) as a one-step method on the first time step. This is accomplished by replacing the right-hand side of (4.11) by (4.13) and choosing $\theta=1 / 2, \xi=0$.

The numerical differentiation formulas (7.4) and (7.5) are exact (i.e., the truncation error is zero) for a polynomial of degree not exceeding three and (7.6) is exact for a polynomial of degree not exceeding two. Since the exact solution (7.2) is a quadratic polynomial of degree two in each spatial variable, the numerical solution of an unfactored algorithm would have the peculiar property that there would be no spatial discretization error. Consequently, the error in a numerical solution for the example problem (7.1) consists of the temporal discretization error and the cross-product error term from the approximate factorization (see, e.g., Eq. (4.4)), and, of course, roundoff error.

For each numerical experiment, we compute the $\mathrm{L}_{2}$ norm of the error which is defined as follows. At a given time, $t=t^{n}=n \Delta t$, the error $e_{j, k}$ at each grid point is defined by

$$
e_{j, k}=u_{j, k}^{n}-u\left(j \Delta x, k \Delta y, t^{n}\right),
$$


where $u_{j, k}^{n}$ is the numerical solution and $u\left(j \Delta x, k \Delta y, t^{n}\right)$ is the analytical solution. The Euclidean or $\mathrm{L}_{2}$ norm of the error is defined by

$$
L_{2} \text { error }=\left[\left(\sum_{j=1}^{J} \sum_{k=1}^{K} e_{j, k}^{2}\right) / J K\right]^{1 / 2}
$$

where $J$ and $K$ are the total number of grid points in the $x$ - and $y$-directions.

The second-order backward differentiation method $(\theta=1, \xi=1 / 2)$ (see

Table 1) was chosen as the generating LMM for the first computational experiment. The $\mathrm{L}_{2}$ errors for the $\Lambda, \Delta$, and $\delta^{2}$ formulations (algorithms (4.12), (5.11), and (5.14)) are shown in Table 2. Each computation was carried out to a given time $(t=1.0)$ with a fixed ratio of $\Delta t / \Delta x=1.0$. The computations were repeated with successively smaller values of $\Delta t$ so that the $\mathrm{L}_{2}$ rate could be computed. (The $\mathrm{L}_{2}$ rate is the slope of a $\log -10 \mathrm{~g}$ graph of the $\mathrm{L}_{2}$ error vs. $\Delta t$. For a second-order method without round-off error, the $L_{2}$ rate should approach two as $\Delta t \rightarrow 0$. ) The results show the second-order accuracy of the methods. Since the same time-differencing method was used for each computation, the differences in the $L_{2}$ error (for a given $\Delta t$ ) result from the cross-product error of the approximate factorization.

The next numerical experiment demonstrates the detrimental effect on the accuracy if the mixed derivative is treated with first-order accuracy. The errors listed in Table 3 were computed using the $\Lambda$ formulation with the backward differentiation method as the generating LMM. For reference, the results listed under column (1) are repeated from Table 2. The $L_{2}$ errors and rates tabulated under column (2) were obtained using (4.12) but with the right-hand side of (4.11) replaced by (4.13), that is, a first-order temporal treatment for the mixed derivative. The degradation in accuracy is obvious. 
Column (3) of Table 3 shows the deterioration in accuracy when the timedependent coefficients $a, b, c$ are not evaluated at the proper time level. The coefficients should be evaluated at $t *$ defined by (6.7) and hence for the backward differentiation method $(\xi=1 / 2) t^{*}=t^{n}+\Delta t$. In obtaining the $\mathrm{L}_{2}$ errors listed in column (3), the coefficients were evaluated at $t^{\mathrm{n}}$ rather than $t *$ and the loss of accuracy is apparent.

It is important to note for $\xi=0$ that the operator $\Lambda$ defined by (4.5) in the $\Lambda$ formulation becomes

$$
\Lambda u^{n}=\Delta u^{n}
$$

Consequently, the $\Lambda$ and $\Delta$ formulations are identical if $\xi=0$. (Recall that the $\Delta$ algorithm is given by (5.6) with $\alpha=0$.) An advantage of the $\Delta$ formulation is that $u^{n-1}$ is not needed to compute $u^{n+l}$ in the final step (5.6c); hence, the $\Delta$ form generally requires the least amount of storage. On the other hand, the $\Delta$ formulation for $\xi \neq 0$ has a significantly reduced parameter space $(\theta, \xi)$ for unconditional stability when applied to hyperbolic problems (see Sec. 8 and [20, Sec. 9]). Consequently, because the $\Lambda$ and $\Delta$ formulations are identical for $\xi=0$, this subclass of schemes has the simplicity of the $\Delta$ form and the robustness of the $\Lambda$ form. Table 4 compares the $\mathrm{L}_{2}$ error and rate for several schemes with $\xi=0$. For a fixed value of $\xi$ in the region of $A_{0}$-stability (see Fig. 1), the error constant is a monotone increasing function of $\theta$. This is verified by comparing the $L_{2}$ errors for a given value of $\Delta t$ in Table 4 .

According to the stability analysis of Appendix $B$, methods that fall in the region between the curves (4.15) are not unconditionally stable in the $\Lambda$ formulation (4.11) for all values of the coefficient $b$ satisfying inequality (1.1d). The last numerical experiment verifies this result for the LMM 
methods 1isted in Table 1 . The parameters used in the computation are listed in the caption of Table 5. The loss of unconditional stability for the trapezoidal formula and Lees method is apparent from the large error for these two methods listed in Table 5.

8. Concluding remarks. In this paper we combine the class of all $A_{0}$-stable second-order linear two-step methods and the method of approximate factorization to construct unconditionally stable ADI methods for parabolic equations (in two space dimensions) with a mixed derivative. For computational simplicity the mixed derivative is treated explicity.

In the application of the approximate factorization method we consider several different solution variables. In Sec. 4 we follow [20] and select $\rho(E) u^{n}$ as the unknown variable. This choice provides a natural framework for constructing unconditionally stable ADI methods for parabolic PDEs by combining $A_{0}$-stable LMMs with approximate factorization. The choice of the unknown variable is not unique and for completeness we derive a general two-step ADI scheme with $(\Delta-\alpha \nabla) u^{n}$ as the unknown variable in Sec. 5. The general formulation contains a parameter $\alpha$ in addition to the parameters $(\theta, \xi)$ of the second-order, two-step method. The parameter space $(\alpha, \theta, \xi)$ for unconditional stability is determined in Appendix B. Several general observations can be made regarding the stability of these schemes: (1) For a given value of $\alpha$ in the range $[-1,1]$, the parameter space $(\theta, \xi)$ for unconditional stability is reduced from that of the unfactored implicit algorithm (3.2) (compare Fig. 1 with Figs. 3 and 4 ), but is increased from that of the unfactored implicitexplicit (i.e., explicit treatment of mixed derivative) algorithm (3.12) (compare Fig. 2 with Figs. 3 and 4). (2) For any allowed value of $\alpha$, the reduced parameter space excludes the familiar (one-step) trapezoidal formula 
and the Lees type scheme (see Table 1). (3) The $\delta^{2} u$ formulation retains the largest parameter space for unconditional stability. (4) The $\rho(E)$ or $\Lambda$ formulation has the peculiar property that $\alpha=\xi /(1+\xi)$, that is, $\alpha$ depends on the particular LMM chosen. The extent of the parameter space for unconditional stability (Fig. 3) is nearly as large as for the $\delta^{2}$ formulation (Fig. 4 with $\alpha=1$ ). (5) Although not considered in this paper, it can be shown that if the general $(\Delta-\alpha \nabla)$ formulation is applied to the model convection equation

$$
\frac{\partial u}{\partial t}=-c_{1} \frac{\partial u}{\partial x}-c_{2} \frac{\partial u}{\partial y}
$$

then only the $\rho(E)$ formulation (i.e., $\alpha=\xi /(1+\xi)$ retains the same parameter space (shaded region of Fig. 1) for unconditional stability as the generating A-stable LMM. In fact, the $\delta^{2}$ formulation has no parameter values $(\theta, \xi)$ for which the scheme is unconditionally stable.

The emphasis of this paper is on the construction of unconditionally stable second-order accurate ADI methods for the model parabolic equation (1.1). By following the approach outlined herein (i.e., the use of $A_{0}$-stable LMMs in conjunction with the method of approximate factorization) one can easily construct algorithms for multidimensional nonlinear parabolic systems. For some auspicious reason, the parameter space $(\theta, \xi)$ for which the class of second-order two-step methods is $A_{0}$-stable happens to coincide with the parameter space for which this class of methods is A-stable. Consequently, one can use the class of time-differencing schemes of this paper to design second-order ADI algorithms for mixed hyperbolic-parabolic systems of nonlinear equations. A noniterative algorithm in the $\Delta$-form for nonlinear systems was considered in [1] and a general development for the $\rho(E)$ formulation is in a companion paper [21]. 
Appendix A. Stability analysis of combined LMMs. In this appendix we examine the stability of the combined LMM (3.7) applied to the model split ODE :

(A.1a,b) $\quad \frac{\mathrm{du}}{\mathrm{dt}}=\lambda_{1} \mathrm{u}+\lambda_{2} \mathrm{u} ; \quad \lambda_{1}<0, \lambda_{1}+\lambda_{2} \leq 0$,

where $\lambda_{1}$ and $\lambda_{2}$ are real constants. In addition, we investigate the stability of the unfactored scheme (3.12) for the PDE (1.1). The analysis is for the class of all second-order, two-step methods.

Consider the combined LMM (3.7) where $\rho(E), \sigma(E)$, and $\sigma_{e}(E)$ are defined by $(2.7 a),(2.9)$, and (3.15). If we apply this scheme to the model equation (A.1) with $\mathrm{f}_{1}=\lambda_{1} \mathrm{u}$ and $\mathrm{f}_{2}=\lambda_{2} \mathrm{u}$, we obtain a difference equation whose characteristic equation is

$$
a_{2} \zeta^{2}+a_{1} \zeta+a_{0}=0
$$

where

(A. 3a)

$$
a_{2}=(1+\xi)-\theta \lambda_{1} \Delta t
$$

$$
a_{1}=-(1+2 \xi)-\left(\xi-2 \theta+\frac{3}{2}\right) \lambda_{1} \Delta t-\left(\xi+\frac{3}{2}\right) \lambda_{2} \Delta t,
$$

(A. 3c)

$$
a_{0}=\xi+\left(\xi-\theta+\frac{1}{2}\right) \lambda_{1} \Delta t+\left(\xi+\frac{1}{2}\right) \lambda_{2} \Delta t
$$

Equation (A.2) is a von Neumann polynomial [16], that is, $|\zeta| \leq 1$, if and only if

(A. 4a)

$$
a_{0} \leq a_{2}
$$

and

$$
-\left(a_{2}+a_{0}\right) \leq a_{1} \leq a_{2}+a_{0}
$$

where without loss of generality $a_{2}$ is assumed to be positive. The inequalities (A.1b) and (A.4) lead to the following conditions for the stability of the combined two-step scheme: 
(A. $5 \mathrm{a}, \mathrm{b}) \quad \xi \geq-\frac{1}{2}, \quad 0 \leq(1+2 \xi)+(1-2 \theta+\xi) \lambda_{1} \Delta t+(1+\xi) \lambda_{2} \Delta t$.

In particular, the conditions for $A_{0}$-stability are

$$
\text { (A. 6a, b) } \quad \xi \geq-\frac{1}{2}, \quad \lambda_{2} \geq-\frac{(1-2 \theta+\xi)}{1+\xi} \lambda_{1} \text {. }
$$

Note that for the special case $\lambda_{2}=0$, (A.6b) becomes $\xi \leq 2 \theta-1$ and conditions (A.6) reduce to $(2.16)$.

For the stability analysis of the unfactored scheme (3.12) for the PDE (1.1) we need only consider the stability of the linear two-step scheme (3.7) applied to the ODE (3.4) for the Fourier coefficient. In this appendix we consider only the spatially continuous solution; however, the results are applicable to the spatially discrete case (see last paragraph of Appendix B). The conditions on the parameters $(\theta, \xi)$ for the unconditional stability of (3.12) can be derived from the $A_{0}$-stability requirements (A.6) and the relations

$$
\text { (A. } 7 \mathrm{a}, \mathrm{b}) \quad \lambda_{1}=-\left(\mathrm{ak}_{1}{ }^{2}+\mathrm{ck}_{2}{ }^{2}\right), \quad \lambda_{2}=-\mathrm{b} \kappa_{1} \kappa_{2},
$$

obtained by comparing (A.1) and (3.4b). There follows

$$
\text { (A. 8a,b) } \quad \xi \geq-\frac{1}{2}, \quad-\mathrm{b}_{1} \kappa_{2} \geq \frac{(1-2 \theta+\xi)}{(1+\xi)}\left(\mathrm{ak}_{1}{ }^{2}+\mathrm{ck}_{2}{ }^{2}\right) \text {. }
$$

The inequalities (A.8a,b) together with (1.1c,d) imply

$$
\text { (A. } 9 \mathrm{a}, \mathrm{b}) \quad \xi \geq-\frac{1}{2}, \quad \xi \leq \theta-1 \text {. }
$$

Inequality (A.9b) is more restrictive than the inequality (2.16a) for the generating two-step method $(2.6)$ to be $A_{o}$-stable. Consequently, we have the result that the second-order explicit treatment of the mixed derivative reduces the parameter space $(\theta, \xi)$ for which the unfactored scheme $(3.12)$ is unconditionally stable (see Fig. 2). 
Appendix B. Stability analysis for two-step ADI schemes. In this appendix we perform a linear stability analysis for the factored scheme (5.5). We assume that $u^{n}$ is spatially continuous and seek a solution of the form

$$
u^{n}=v^{n} e^{i\left(\kappa_{1} x+\kappa_{2} y\right)}
$$

where $v^{n}$ is the Fourier coefficient and $\kappa_{1}, \kappa_{2}$ are the Fourier variables. Prior to an actual numerical computation, the spatial derivatives are replaced by appropriate difference quotients; however, as indicated at the end of this appendix, the stability proof for the spatially discrete case requires only a minor modification of the following stability proof.

By substituting (B.1) into (5.5), we find that the Fourier coefficient satisfies

$$
\text { (B.2) } \begin{aligned}
(1+A)(1+C)(\Delta-\alpha \nabla) v^{n}= & -\frac{1}{\theta}(A+B+C)\left[1+\left(\xi-\theta+\frac{1}{2}\right) \nabla\right] v^{n} \\
& -B \nabla v^{n}-\alpha(A+C) \nabla v^{n}+\left(\frac{\xi}{1+\xi}-\alpha\right) \nabla v^{n},
\end{aligned}
$$

where we have defined

$$
A=\omega \Delta \operatorname{tak}_{1}{ }^{2}, \quad B=\omega \Delta t b \kappa_{1} \kappa_{2}, \quad C=\omega \Delta t c \kappa_{2}^{2}
$$

and $\omega=\theta /(1+\xi)$. The amplification factor is defined by

$$
v^{n+l}=\zeta v^{n}
$$

and consequently it follows from (B.2) that $\zeta$ satisfies the quadratic equation

$$
a_{2} \zeta^{2}+a_{1} \zeta+a_{0}=0
$$

where

(B.6a)

$$
\begin{aligned}
a_{2}= & (1+A)(1+C), \\
a_{1}= & -(1+\alpha)(1+A)(1+C)+\frac{1}{\theta}\left(\xi-\theta+\frac{3}{2}\right)(A+B+C) \\
& +B+\alpha(A+C)-\left(\frac{\xi}{1+\xi}-\alpha\right),
\end{aligned}
$$


(B. 6c)

$$
a_{0}=\alpha(1+A)(1+C)-\frac{1}{\theta}\left(\xi-\theta+\frac{1}{2}\right)(A+B+C)
$$

$$
-B-\alpha(A+C)+\left(\frac{\xi}{1+\xi}-\alpha\right) \text {. }
$$

In the one-dimensional case $(b=c=0$ in Eq. (1.1) and $B=C=0$ in (B.5)), the roots of the quadratic (B.5) have modulus bounded by unity for those values of $(\theta, \xi)$ shown in the shaded region of Fig. 1 , that is,

$$
\text { (B. } 7 a, b) \quad \xi \leq 2 \theta-1, \quad \xi \geq-\frac{1}{2} .
$$

This one-dimensional result follows from the analysis [20]. Note that $\alpha$ only enters as a parameter in the two-dimensional factored algorithm (5.5). (Recall that (5.2) and (5.3) are actually identical.) One can easily verify that the coefficients (B.6) do not depend on $\alpha$ if $B=C=0$. We must determine if there are additional restrictions on the parameters $(\theta, \xi)$ for the unconditional stability of the factored scheme (5.5) for arbitrary values of $a, b, c$ subject only to the parabolicity conditions

$$
\text { (B. } 8 \mathrm{a}, \mathrm{b}) \quad \mathrm{a}>0, \mathrm{~b}^{2}<4 \mathrm{ac}
$$

of the partial differential equation (1.1). Since the one-dimensional problem $(b=c=0)$ is a special case of the two-dimensional problem, we need not consider values of $(\theta, \xi)$ outside the domain (B.7). Hence $\omega>0, A$ and $C$ as defined by (B. 3) are positive, and

$$
\mathrm{A}+\mathrm{B}+\mathrm{C}=\omega \Delta \mathrm{t}\left(\mathrm{ak}_{1}{ }^{2}+\mathrm{b} \kappa_{1} \mathrm{~K}_{2}+\mathrm{ck}_{2}{ }^{2}\right)>0
$$

since the positive definiteness of this quadratic form was the condition which led originally to (B. 8).

The coeffictents (B.6) of the quadratic (B.5) are real and consequently the roots $\zeta$ satisfy $|\zeta| \leq 1$ if and only if the inequalities (A.4) of Appendix $A$ are satisfied. If we insert $a_{0}$ and $a_{2}$ as given by (B.6) into (A. 4a), there follows 


$$
0 \leq \frac{1}{1+\xi}+(1-\alpha) A C+\frac{1}{\theta}\left(\xi+\frac{1}{2}\right)(A+B+C)
$$

which is satisfied for all allowable A,B,C if and only if

$\alpha \leq 1$.

(Recall that the parameters $\theta$ and $\xi$ are required to satisfy inequalities (B.7).) Likewise the left inequality of (A.4b) is satisfied. If the coefficients (B.6) are inserted into the right inequality of (A.4b) one obtains

$$
0 \leq(1+\alpha) A C-\frac{1}{\omega} B+\frac{1+2 \xi}{1+\xi}+\left(2-\frac{1}{\omega}\right)(A+C) .
$$

The determination of necessary and sufficient conditions for this inequality is simplified if it is rewritten as

$$
\text { (B. 13) } 0 \leq(1+\alpha) a c\left(k_{1} k_{2}\right)^{2}-\frac{1}{\omega} b k_{1} k_{2}+\frac{1+2 \xi}{1+\xi}+\left(2-\frac{1}{\omega}\right)\left(a k_{1}{ }^{2}+c k_{2}{ }^{2}\right)
$$

where we have used the definitions (B. 3) and defined:

$$
\text { (B. 14a,b) } \quad k_{1}=\sqrt{\omega \Delta t} k_{1}, \quad k_{2}=\sqrt{\omega \Delta t} k_{2} .
$$

It can be shown that necessary and sufficient conditions for the polynomial $P$ in $k_{1}, k_{2}$ defined by

$$
P=e_{1}\left(k_{1} k_{2}\right)^{2}+e_{2} k_{1} k_{2}+e_{3}+e_{4} k_{1}^{2}+e_{5} k_{2}^{2}
$$

to be positive semidefinite (i.e., $\mathrm{P} \geq 0$ for all real $\mathrm{k}_{1}, \mathrm{k}_{2}$ ) are

$$
e_{1}, e_{3}, e_{4}, e_{5} \geq 0 \text {, }
$$

$$
\left|e_{2}\right| \leq 2 \sqrt{e_{1} e_{3}}+2 \sqrt{e_{4} e_{5}} .
$$

Comparison of (B.15) and (B.13) leads to the conditions

$$
\begin{array}{ll}
\text { (B. } 17 a, b) & (1+\alpha) a c \geq 0, \\
\text { (B. 17c,d } \quad & \left(2-\frac{1}{\omega}\right) a \geq 0, \quad\left(2-\frac{1}{\omega}\right) c \geq 0,
\end{array}
$$

(B. 17e)

$$
\left|\frac{b}{\omega}\right| \leq 2 \sqrt{(1+\alpha) a c \frac{1+2 \xi}{1+\xi}}+2 \sqrt{\left(2-\frac{1}{\omega}\right)^{2} \text { ac }} \text {. }
$$


Inequalities (B.17a,b,c,d) are satisfied by virtue of inequalities (B.7), (B. $8 \mathrm{a})$, and (B.11) plus the constraint: $-1 \leq \alpha$

Inequality (B.17e) can be rewritten as

$$
\frac{1}{w}|b| \leq 2 \sqrt{\mathrm{ac}}\left[\sqrt{\frac{(1+\alpha)(1+2 \xi)}{1+\xi}}+\left(2-\frac{1}{\omega}\right)\right],
$$

which is satisfied for all allowable $a, b, c$ (see inequalities (B.8)) if and only if

$$
\frac{1}{\omega} \leq \sqrt{\frac{(1+\alpha)(1+2 \xi)}{1+\xi}}+\left(2-\frac{1}{\omega}\right) \text {, }
$$

or

$$
\theta \geq \frac{2(1+\xi)}{2+\sqrt{\frac{(1+\alpha)(1+2 \xi)}{1+\xi}}}
$$

Hence the final inequalities which must be satisfied are (B. $7 \mathrm{~b}),($ B.11), (B.13), and (B.21).

In the above stability analysis we assumed that the spatial derivatives were continuous. Since in practice the spatial derivatives are replaced by discrete difference quotients, it remains to consider the spatially discrete case. If, for example, the spatial derivatives in (5.5) are replaced by the second-order difference quotients $(7.4)-(7.6)$, then the stability analysis proceeds as above with the exception that the exponential in (B.1) is replaced by

$$
u_{j, k}^{n}=v^{n} e^{i\left(k_{1} j \Delta x+k_{2} k \Delta y\right)},
$$

where $x=j \Delta x, y=k \Delta y$. If we make the following correspondence

$$
\text { (B. 23a,b) } \quad \kappa_{1}+\frac{2 \sin \left(\theta_{1} / 2\right)}{\Delta x}, \quad \kappa_{2}+\frac{2 \sin \left(\theta_{2} / 2\right)}{\Delta y} \text {, }
$$




$$
B+B \cos \frac{\theta_{1}}{2} \cos \frac{\theta_{2}}{2},
$$

where

$$
\theta_{1}=\kappa_{1} \Delta \mathrm{x}, \quad \theta_{2}=\kappa_{2} \Delta \mathrm{y},
$$

between the parameters for the discrete and continuous case, then the amplification factor for the discrete case satisfies the same quadratic (B.5) with coefficients (B.6). Since the stability region defined by inequalities (B.7b), (B.11), (B.18), and (B.21) is valid for arbitrary values of $\kappa_{1}$ and $\kappa_{2}$ and

$$
\left|B \cos \frac{\theta_{1}}{2} \cos \frac{\theta_{2}}{2}\right| \leq|B|,
$$

we obtain the same stability range for the discrete case. If one uses a noncentered approximation for the mixed derivative such as

$$
\left.\frac{\partial^{2} Q}{\partial x \partial y}\right|_{j, k}=\frac{1}{2 \Delta x \Delta y}\left[\nabla_{x} \Delta_{y}+\Delta_{x} \nabla_{y}\right] Q_{j, k}+0\left(\Delta x^{2}, \Delta y^{2}\right),
$$

$$
\begin{aligned}
= & \frac{1}{2 \Delta x \Delta y}\left(Q_{j+1, k}-2 Q_{j, k}+Q_{j-1, k}-Q_{j+1, k-1}\right. \\
& \left.+Q_{j, k+1}+Q_{j, k-1}-Q_{j-1, k+1}\right)+0\left(\Delta x^{2}, \Delta y^{2}\right),
\end{aligned}
$$

where

$$
\Delta_{x} Q_{j}=Q_{j+1}-Q_{j}, \quad \nabla_{x} Q_{j}=Q_{j}-Q_{j-1}, \quad \text { etc. }
$$

the only modification necessary in the stability analysis is replacement of (B. 24) by

$$
B \leftarrow B \cos \left(\frac{{ }^{\theta} x-\theta y}{2}\right)
$$




\section{REFERENCES}

[1] R. M. BEAM and R. F. WARMING, An implicit factored scheme for the compressible Navier-Stokes equations, AIAA J., 16 (1978), pp. 393-402.

[2] M. CIMENT, S. H. LEVENTHAL, and B. C. WEINBERG, The operator compact implicit method for parabolic equations, J. Computational Phys., 28 (1978), pp. 135-166.

[3] C. W. CRYER, A new class of highly-stable methods: Ao-stable methods, BIT, 13 (1973) pp. 153-159.

[4] G. DAHLQUIST, A special stability problem for linear multistep methods, BIT, 3 (1963), pp. 27-43.

[5] __ Error analysis for a class of methods for stiff non-linear initial value problems, Numerical Analysis Dundee 1975, Lecture Notes in Mathematics 506, A. Dold and B. Eckmann, eds., Springer-Verlag, Berlin, 1976, pp. 60-72.

[6] _ The theory of linear multistep methods and related mathematical topics, Lecture Notes (microfilm), Dept. of Numerical Analysis, Royal Inst. of Tech., Stockholm, 1976.

[7] _ Positive functions and some applications to stability questions for numerical methods, preprint, MRC Symposium, Madison, Wisc., April 1978. Proceeding to be published by Academic Press.

[8] J. DOUGLAS, On the numerical integration of $u_{x x}+u_{y y}=u_{t}$ by implicit methods, J. Soc. Indust. App1. Math., 3 (1955), pp. 42-65.

[9] J. DOUGLAS and J. E. GUNN, A general formulation of alternating direction methods, Numer. Math., 6 (1964), pp. 428-453.

[10] C. W. GEAR, Numerical Initial Value Problems in Ordinary Differentia1 Equations, Prentice-Ha11, Englewood Cliffs, N.J., 1971. 
[11] E. ISAACSON and H. B. KELLER, Analysis of Numerical Methods, John Wiley and Sons, New York, 1966.

[12] S. R. K. IYENGAR and M. K. JAIN, Comparative study of two and three level ADI methods for parabolic equations with a mixed derivative, Int. Journal for Numerical Methods in Eng., 10 (1976), pp. 1309-1315.

[13] P. D. LAX and R. D. RICHTMYER, Survey of the stability of linear finite difference equations, Comm. Pure App1. Math., 9 (1956), pp. 267-293.

[14] M. LEES, A linear three-level difference scheme for quasilinear parabolic equations, Math. Comp., 20 (1966), pp. 516-522.

[15] S. MCKEE and A. R. MITCHELL, Alternating direction methods for parabolic equations in two space dimensions with a mixed derivative, Computer Journal, 13 (1970), pp. 81-86.

[16] J. J. H. MILLER, On the location of zeros of certain classes of polynomials with applications to numerical analysis, J. Inst. Maths. Applics., 8 (1971), pp. 397-406.

[17] 0. NEVANLINNA and W. LINIGER, Contractive methods for stiff differential equations, BIT, 18 (1978), pp. 457-474.

[18] D. W. PEACEMAN and H. H. RACHFORD, The numerical solution of parabolic and elliptic differential equations, J. Soc. Indust. App1. Math., 3 (1955), pp. 28-41.

[19] R. F. WARMING and R. M. BEAM, On the construction and application of implicit factored schemes for conservation laws, Symposium on Computational Fluid Dynamics, New York, April 16-17, 1977; SIAM-AMS Proceedings, 11 (1978), pp. 85-129. 
[20] R. F. WARMING and R. M. BEAM, An extension of A-stability to alternating direction implicit methods, submitted to BIT. Also NASA TM-78537, 1978.

[21] R. F. WARMING and R. M. BEAM, Factored, A-stable, linear multistep methods - an alternative to the method of lines for multidimensions, Conference Proceedings, 1979 SIGNUM Meeting on Numerical ODE's, Champaign, I11., Apri1 3-5, 1979. 
TABLE 1

Partial 1ist of second-order two-step methods.

\begin{tabular}{c|c|c|l|c}
\hline$\theta$ & $\xi$ & $\phi$ & Method & $\begin{array}{c}\text { Symbol in } \\
\text { Fig. 1 }\end{array}$ \\
\hline$\frac{1}{2}$ & 0 & 0 & One-step trapezoidal formula & $\bullet$ \\
1 & $\frac{1}{2}$ & 0 & Backward differentiation & $\bullet$ \\
$\frac{1}{3}$ & $-\frac{1}{2}$ & $-\frac{1}{3}$ & Lees type [14] & $\bullet$ \\
$\frac{3}{4}$ & 0 & $-\frac{1}{4}$ & Adams type [17] & $\bullet$ \\
$\frac{1}{2}$ & $-\frac{1}{2}$ & $-\frac{1}{2}$ & Two-step trapezoidal formula & । \\
\hline
\end{tabular}


TABLE 2

$L_{2}$ error of the $\Lambda, \Delta$, and $\delta^{2}$ formulations at $t=1.0$.

\begin{tabular}{|c|c|c|c|c|c|c|c|c|}
\hline \multirow{2}{*}{$\begin{array}{c}\Delta t= \\
\Delta x=\Delta y\end{array}$} & \multirow{2}{*}{$\begin{array}{l}\text { Number of } \\
\text { time steps }\end{array}$} & \multirow{2}{*}{$\Delta t / \Delta x^{2}$} & \multicolumn{2}{|c|}{$\Lambda$ formulation } & \multicolumn{2}{|c|}{$\Delta$ formulation } & \multicolumn{2}{|c|}{$\delta^{2}$ formulation } \\
\hline & & & $\mathrm{L}_{2}$ error & $\mathrm{L}_{2}$ rate & $\mathrm{L}_{2}$ error & $\mathrm{L}_{2}$ rate & $L_{2}$ error & $\mathrm{L}_{2}$ rate \\
\hline 0.2 & 5 & 5 & $0.785 \times 10^{-2}$ & \multirow{4}{*}{$\begin{array}{l}2.02 \\
2.01 \\
2.01\end{array}$} & $0.107 \times 10^{-1}$ & \multirow{4}{*}{$\begin{array}{l}2.01 \\
2.03 \\
2.03\end{array}$} & $0.134 \times 10^{-2}$ & \multirow{4}{*}{$\begin{array}{l}1.57 \\
1.72 \\
1.89\end{array}$} \\
\hline 0.1 & 10 & 10 & $0.193 \times 10^{-2}$ & & $0.266 \times 10^{-2}$ & & $0.452 \times 10^{-3}$ & \\
\hline 0.05 & 20 & 20 & $0.479 \times 10^{-3}$ & & $0.649 \times 10^{-3}$ & & $0.137 \times 10^{-3}$ & \\
\hline 0.025 & 40 & 40 & $0.119 \times 10^{-3}$ & & $0.160 \times 10^{-3}$ & & $0.372 \times 10^{-4}$ & \\
\hline
\end{tabular}


TABLE 3

Numerical experiments illustrating (1) second-order $\Lambda$ formulation, (2) degradation in accuracy when mixed derivative is computed with a first-order method, (3) deterioration in accuracy when time-dependent coefficients are not evaluated at proper time level.

\begin{tabular}{|c|c|c|c|c|c|c|c|c|}
\hline$\Delta t=$ & Number of & & (1) & & (2) & & (3) & \\
\hline$\Delta \mathrm{x}=\Delta \mathrm{y}$ & time steps & $\Delta\left[/ \Delta x^{-}\right.$ & $\mathrm{L}_{2}$ error & $\mathrm{L}_{2}$ rate & $\mathrm{L}_{2}$ error & $\mathrm{L}_{2}$ rate & $\mathrm{L}_{2}$ error & $\mathrm{L}_{2}$ rate \\
\hline 0.2 & 5 & 5 & $0.758 \times 10^{-2}$ & \multirow{4}{*}{$\begin{array}{l}2.02 \\
2.01 \\
2.01\end{array}$} & $0.585 \times 10^{-2}$ & \multirow{2}{*}{0.59} & $0.907 \times 10^{-2}$ & \multirow{2}{*}{1.72} \\
\hline 0.1 & 10 & 10 & $0.193 \times 10^{-2}$ & & $0.389 \times 10^{-2}$ & & $0.275 \times 10^{-2}$ & \\
\hline 0.05 & 20 & 20 & $0.479 \times 10^{-3}$ & & $0.216 \times 10^{-2}$ & 0.85 & $0.888 \times 10^{-3}$ & 1.63 \\
\hline 0.025 & 40 & 40 & $0.119 \times 10^{-3}$ & & $0.113 \times 10^{-2}$ & & $0.320 \times 10^{-3}$ & \\
\hline
\end{tabular}


TABLE 4

$L_{2}$ error of the $\Lambda$ formulation for $\xi=0$ and several values of $\theta$ at $t=1.0$.

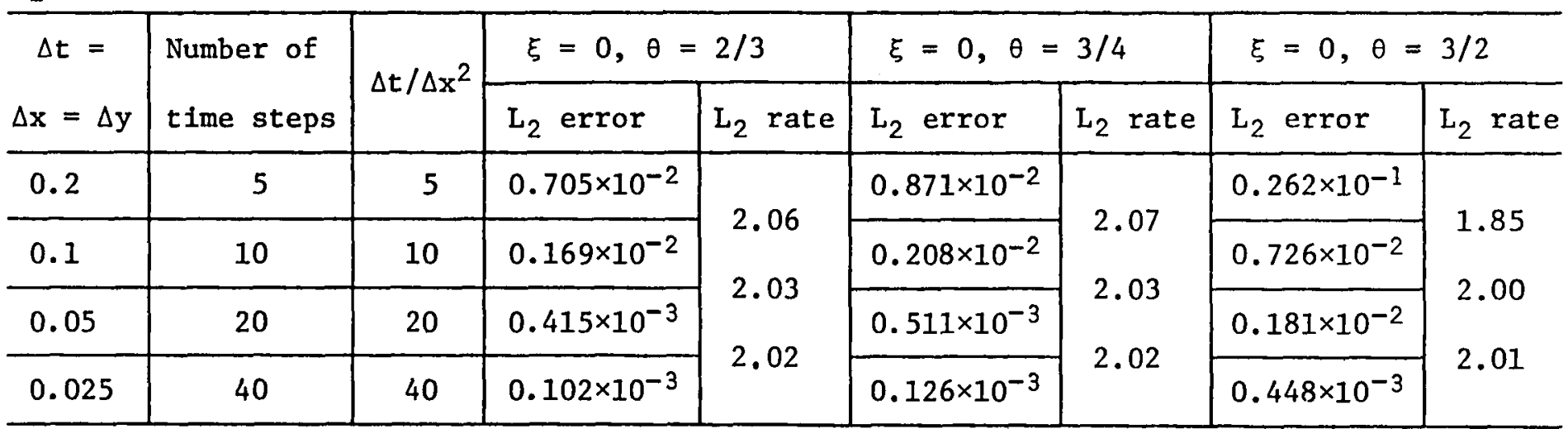




\section{TABLE 5}

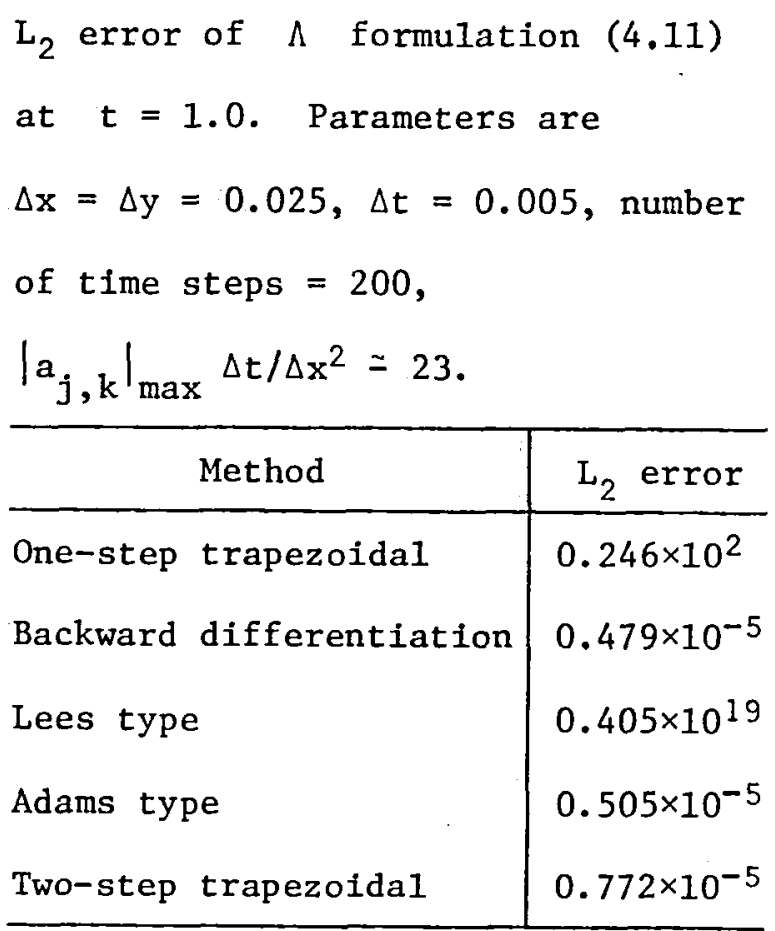




\section{FIGURE CAPTIONS}

FIG. 1. $A_{0}-$ and A-stable domain of the parameters $(\theta, \xi)$ for the class of a11 second-order two-step methods. Symbols denote methods listed in Table 1 .

FIG. 2. Unconditionally stable domain of the parameters $(\theta, \xi)$ for the unfactored scheme (3.12) with $\rho(E), \sigma(E)$, and $\sigma_{e}(E)$ defined by $(2.7 a),(2.9)$, and $(3.15)$.

FIG. 3. Unconditionally stable domain of the parameters $(\theta, \xi)$ for the factored $\Lambda$ formulation (4.11).

FIG. 4. Unconditionally stable domain of the parameters $(\theta, \xi)$ for the factored general formulation (5.5) for several values of $\alpha$. 


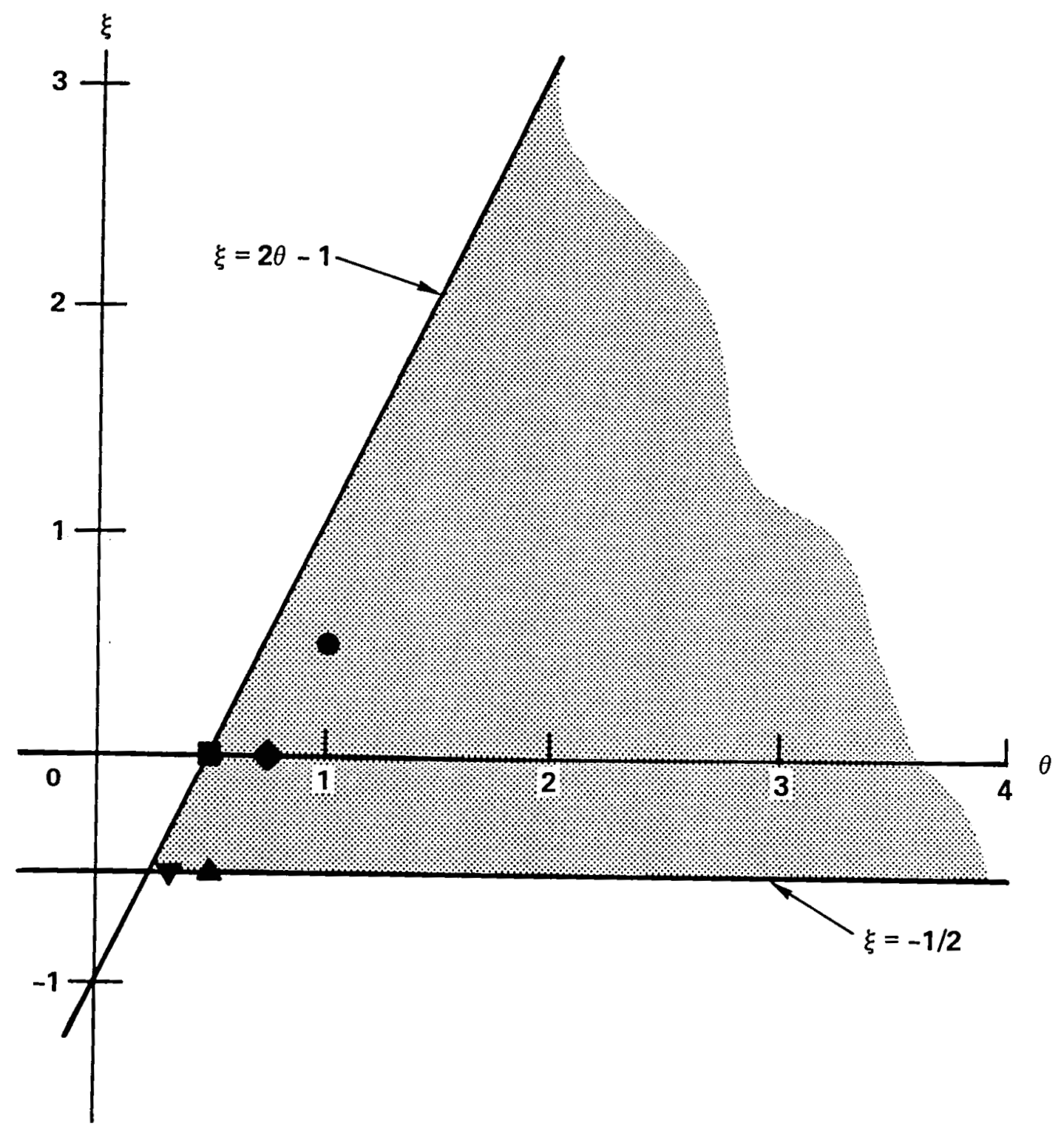

Figure 1.- $A_{0}$ - and A-stable domain of the parameters $(\theta, \xi)$ for the class of all second-order two-step methods. Symbols denote methods listed in Table 1. 


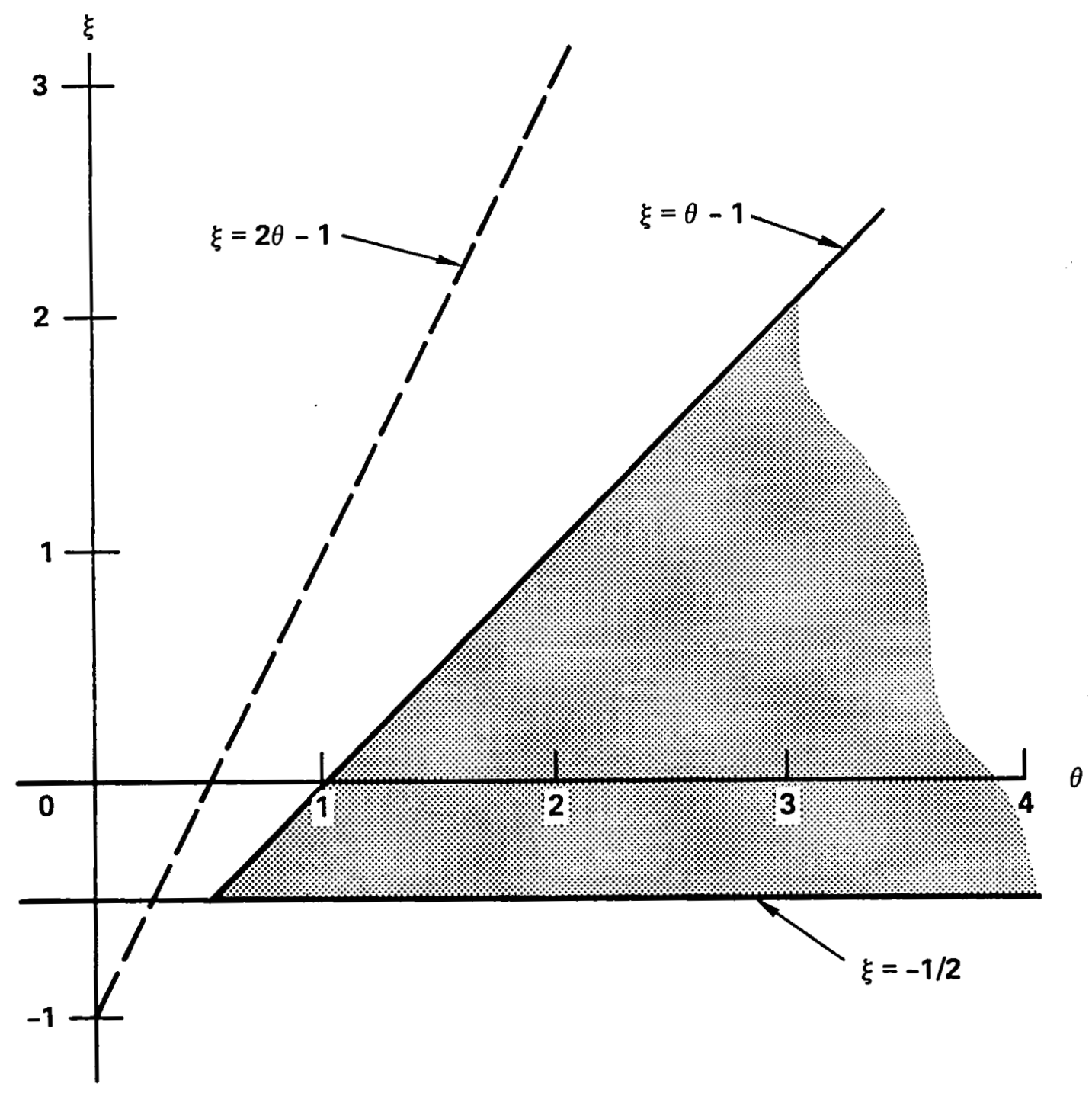

Figure 2.- Unconditionally stable domain of the parameters $(\theta, \xi)$ for the unfactored scheme (3.12) with $\rho(E), \sigma(E)$, and $\sigma_{e}(E)$ defined by $(2.7 a)$, (2.9), and (3.15). 


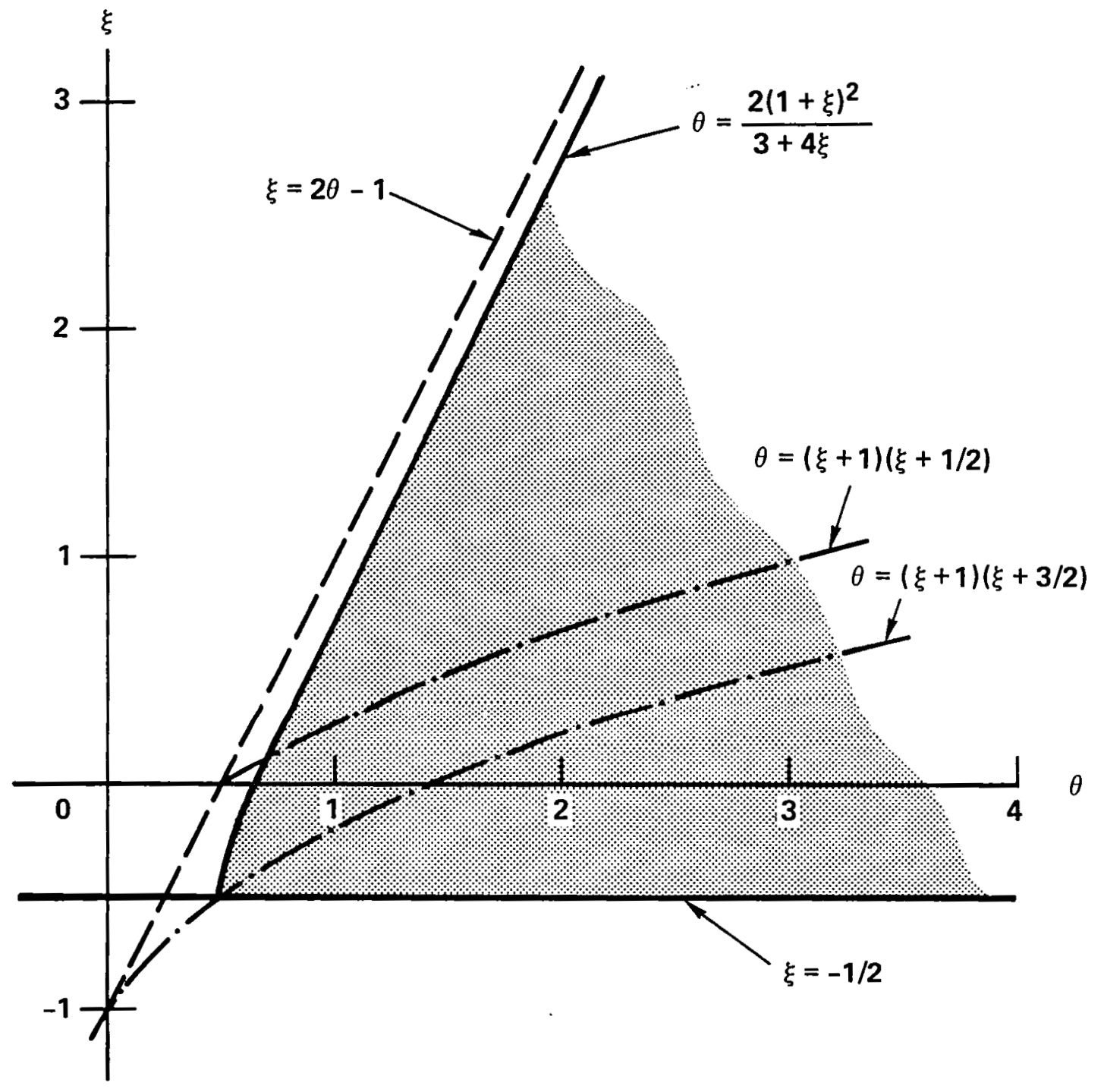

Figure 3.- Unconditionally stable domain of the parameters $(\theta, \xi)$ for the factored $\Lambda$ formulation (4.11). 


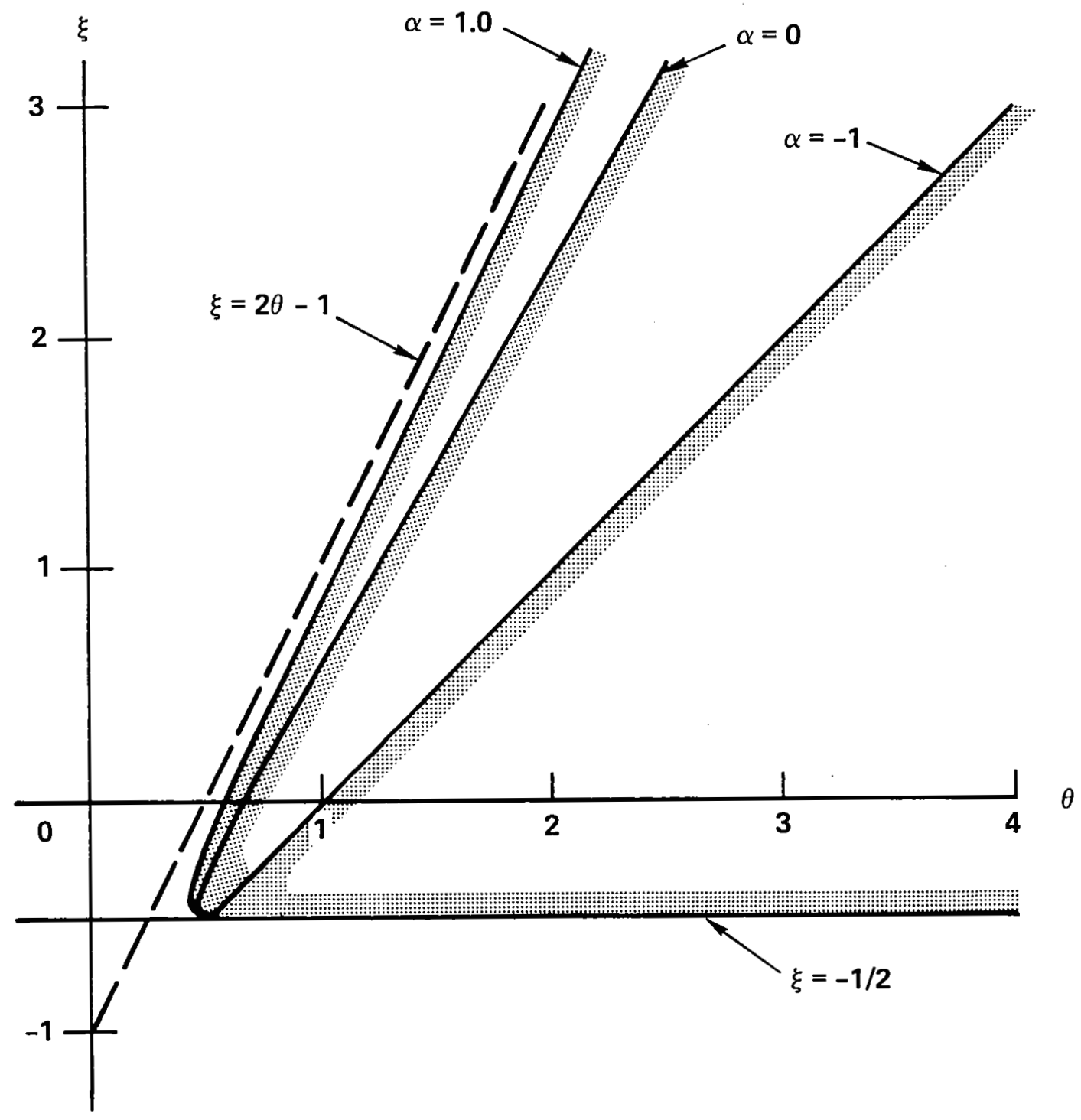

Figure 4.- Unconditionally stable domain of the parameters $(\theta, \xi)$ for the factored general formulation (5.5) for several values of $\alpha$. 


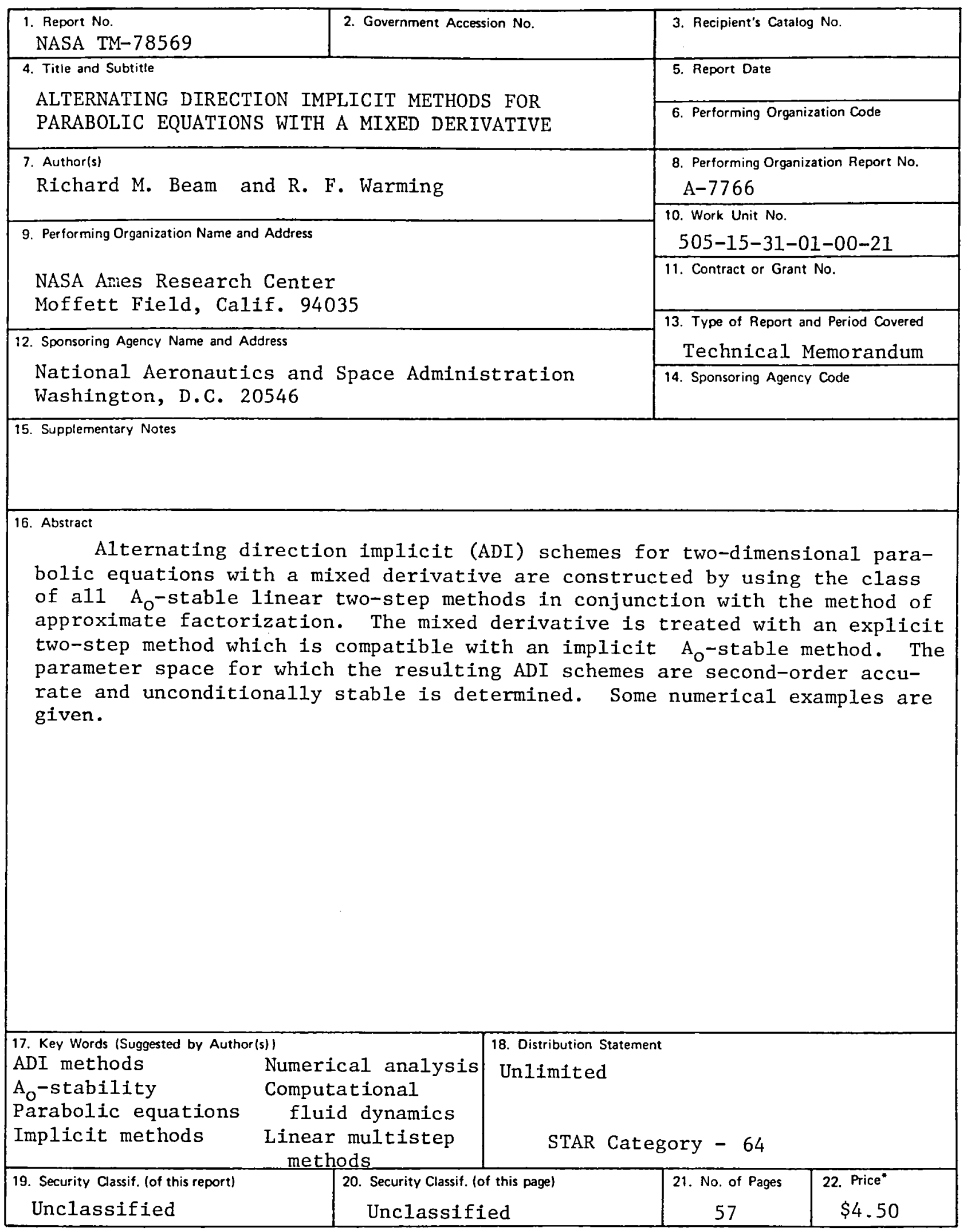

- For sale by the National Terhnical Information Service, Springfield, Virginia 22161 